\title{
THE MAGELLANIC BRIDGE AS A DAMPED LYMAN ALPHA SYSTEM: PHYSICAL PROPERTIES OF COLD GAS TOWARD PKS 0312-770*
}

\author{
Toru Misawa ${ }^{1,2}$, Jane C. Charlton $^{1}$, Henry A. Kobulnicky ${ }^{3}$, Bart P. Wakker ${ }^{4}$, and Joss Bland-Hawthorn ${ }^{5}$

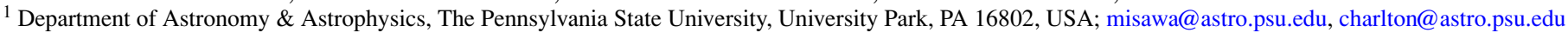 \\ 2 Cosmic Radiation Laboratory, RIKEN, 2-1 Hirosawa, Wako, Saitama 351-0198 Japan \\ ${ }^{3}$ Department of Physics \& Astronomy, University of Wyoming, Laramie, WY 82070, USA; chipk@uwyo.edu \\ ${ }^{4}$ Department of Astronomy, University of Wisconsin-Madison, 475 North Charter Street, Madison, WI 53706, USA; wakker@astro.wisc.edu \\ ${ }^{5}$ School of Physics, University of Sydney, NSW 2006, Australia; jbh@aao.gov.au \\ Received 2008 September 23; accepted 2009 January 31; published 2009 April 7
}

\begin{abstract}
We measure the physical properties of a local multicomponent absorption-line system at $V_{\odot} \sim 200 \mathrm{~km} \mathrm{~s}^{-1}$ toward the quasar PKS 0312-770 behind the Magellanic Bridge (MB) using Hubble Space Telescope Space Telescope Imaging Spectrograph (STIS) spectroscopy in conjunction with photoionization modeling. At an impact parameter of $\sim 10 \mathrm{kpc}$ from the Small Magellanic Cloud (SMC), this sightline provides a unique opportunity to probe the chemical properties and ionization structure in a nearby absorption line system with a column density of log $N_{\mathrm{H} \text { I }}$ $\sim 20.2$, at the transition between damped Ly $\alpha$ (DLA) and sub-DLA systems. We find that metallicity of -1.0 $<\log \left(Z / Z_{\odot}\right)<-0.5$ and ionization parameter of $-6<\log U<-5$ for three low-ionization components and $\log U \sim-2.6$ for one high-ionization component. One component at $V_{\odot}=207 \mathrm{~km} \mathrm{~s}^{-1}$ shows an $\alpha$-element abundance $\log (\mathrm{Si} / \mathrm{H}) \sim-5.0$, making it $\sim 0.2$ dex more metal-rich than both SMC H II regions and stars within the $\mathrm{MB}$ and the SMC. The N/Si ratio in this component is $\log (\mathrm{N} / \mathrm{Si})=-0.3 \pm 0.1$, making it comparable to other $\mathrm{N}$-poor dwarf galaxies and $\sim 0.2$ dex lower than $\mathrm{H}$ II regions in the SMC. Another component at $V_{\odot}=236 \mathrm{~km} \mathrm{~s}-1$ shows a similar $\mathrm{Si} / \mathrm{H}$ ratio but has $\log (\mathrm{N} / \mathrm{Si})=-1.0 \pm 0.2$, indicating a nitrogen deficiency comparable to that seen in the most N-poor DLA systems. These differences imply different chemical enrichment histories between components along the same sightline. Our results suggest that if these absorbers are representative some fraction of DLA systems, then (1) DLA systems along single sightlines do not necessarily represent the global properties of the absorbing cloud, and (2) the chemical composition within a given DLA cloud may be inhomogeneous.
\end{abstract}

Key words: galaxies: abundances - galaxies: individual (Large Magellanic Cloud, Small Magellanic Cloud) Magellanic Clouds - quasars: absorption lines

Online-only material: color figures

\section{INTRODUCTION}

A physical connection between the Large Magellanic Cloud (LMC) and the Small Magellanic Cloud (SMC) was implied as early as 60 years ago (Shapley 1940). A continuous H I gas structure between the LMC and the SMC, known as the Magellanic Bridge (MB), was first reported by Hindman et al. (1963), and it is found to be at a distance of $\sim 50-60 \mathrm{kpc}$ (e.g., Harries et al. 2003). There is substantial evidence for a young stellar population in the MB, with stars as young as $\sim 20 \mathrm{Myr}$ (Irwin et al. 1985, 1990; Grondin et al. 1992; Hambly et al. 1994; Demers \& Battinelli 1998) and with molecular clouds detected (Lehner 2002; Muller et al. 2003; Mizuno et al. 2006). These young populations were probably formed locally, because they are not old enough to have escaped from the next nearest star-forming region (i.e., the SMC) based on their peculiar motions. On the other hand, the larger-scale tidal structure that is connected to the MB, the Magellanic Stream, contains only gas, with no evidence of a stellar population (Guhathakurta \& Reitzel 1998). The physical connection of these structures is still under discussion (Kallivayalil et al. 2006; Piatek et al. 2008).

Although the formation mechanism of the MB is not understood in detail, gravitational tidal interactions probably played

\footnotetext{
* Based on observations obtained with the NASA/ESA Hubble Space Telescope, which is operated by the Space Telescope Science Institute (STScI) for the Association of Universities for Research in Astronomy, Inc., under NASA contract NAS5D26555.
}

a primary role (e.g., Gardiner et al. 1994; Murai \& Fujimoto 1980). Numerical simulations indicate that the MB was created through a close interaction between the LMC and the SMC about 0.2 Gyr ago (e.g., Gardiner \& Noguchi 1996). Thus, the MB is an ideal target for studies of the influence of dynamical interactions on star-forming activity. The MB also provides a unique opportunity to study nearby star formation in a low-metallicity environment. The MB stars have metallicities of only one-tenth that of normal Population I Galactic stars (i.e., even smaller than the SMC stars by $\sim 0.5$ dex; Rolleston et al. 1999).

The abundance pattern of the MB has been studied through stellar populations and gas clouds that are detected as absorption features in the stellar spectra (e.g., Demers \& Battinelli 1998; Lehner et al. 2001, 2008; Lehner 2002; Mallouris et al. 2001; Welty et al. 2001; Li et al. 2006; Nishiyama et al. 2007; Harris 2007). Recently, Carrera et al. (2008) found a possible metallicity gradient in the SMC toward outer regions from $\sim 1^{\circ}$ to $\sim 4^{\circ}$ from its center. However, stars and circumstellar regions could be biased because their physical conditions are significantly influenced by nearby stars. Analysis of interstellar gas should yield more representative properties of the MB and its abundance pattern.

Quasar absorption lines are powerful tools to investigate the physical conditions of absorbers located along sightlines to quasars. These absorbers are detected in quasar spectra, regardless of the source luminosity, which enables us to collect a homogeneous sample of absorbing clouds from early cosmic 
Table 1

HST/STIS Observation Log

\begin{tabular}{|c|c|c|c|c|c|c|c|c|c|}
\hline (1) & $\begin{array}{c}\text { RA } \\
\text { (h:m:s) } \\
(2)\end{array}$ & $\begin{array}{c}\text { Dec } \\
(\mathrm{d}: \mathrm{m}: \mathrm{s}) \\
(3)\end{array}$ & $\begin{array}{l}z_{\mathrm{em}} \\
(4)\end{array}$ & $\begin{array}{c}m_{V} \\
(\mathrm{mag}) \\
(5)\end{array}$ & $\begin{array}{c}\text { Date } \\
\text { (yyyy mm dd) } \\
(6)\end{array}$ & $\begin{array}{c}\text { Grating } \\
\text { (7) }\end{array}$ & $\begin{array}{c}\lambda \text {-coverage } \\
(\AA) \\
(8)\end{array}$ & $\begin{array}{c}t_{\exp } \\
(\mathrm{sec}) \\
(9)\end{array}$ & HST Dataset ID \\
\hline PKS 0312-770 & $03: 11: 55.4$ & $-76: 51: 50.8$ & 0.2230 & 16.1 & $\begin{array}{lll}2001 & 05 & 12 \\
2001 & 03 & 07 \\
2001 & 10 & 16 \\
2001 & 10 & 14\end{array}$ & $\begin{array}{l}\text { E140M } \\
\text { E140M } \\
\text { E140M } \\
\text { E230M }\end{array}$ & $\begin{array}{l}1150-1729 \\
1150-1729 \\
1150-1729 \\
2132-2984\end{array}$ & $\begin{array}{c}12629 \\
12629 \\
12650 \\
6060\end{array}$ & $\begin{array}{l}\text { O65T01010 } \\
\text { O65T02010 } \\
\text { O65T13010 } \\
\text { O65T14010 }\end{array}$ \\
\hline
\end{tabular}

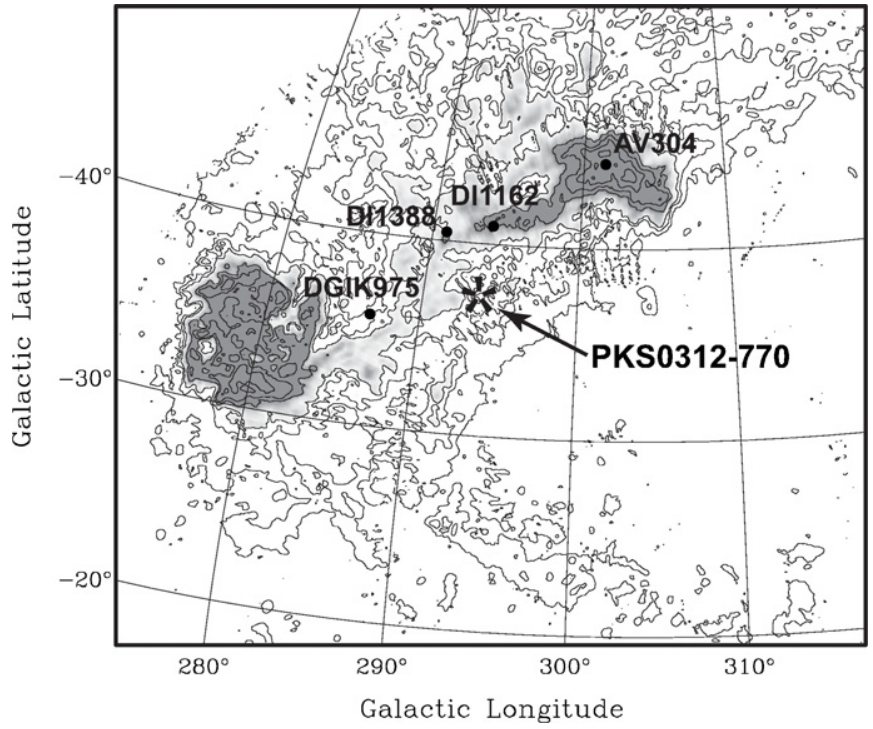

Figure 1. Location of our target, PKS 0312-770, as well as stars in the MB (DGIK 975 and DI 1162, and DI 1388) and the SMC (AV 304), superimposed on the H I peak brightness temperature $\left(T_{B}\right)$ from Putman et al. (1998). Contours mark the $0.1 \mathrm{~K}(5 \sigma), 0.8,2,8,16,32,64$, and $128 \mathrm{~K}$ peak brightness. Light and deep gray contours correspond to $T_{B}>2 \mathrm{~K}$ and $>8 \mathrm{~K}$, respectively. Light gray contour roughly corresponds to the minimum threshold DLA column density, $N_{\mathrm{HI}}=10^{20.3} \mathrm{~cm}^{-2}$ if the total line width of $\mathrm{HI} 21-\mathrm{cm}$ absorption is similar to that toward PKS 0312-770, $d v \sim 50 \mathrm{~km} \mathrm{~s}^{-1}$ (see Figure 5).

epochs to the present. Although there have been several attempts to trace the MB gas along the sightlines toward quasars, these were based on radio spectra that covered $\mathrm{H}_{\mathrm{I}} 21 \mathrm{~cm}$ absorption (e.g., Kobulnicky \& Dickey 1999) or optical spectra that covered the Ca II absorption doublet (Smoker et al. 2005). On the other hand, most important ions (e.g., Mg I, Mg II, Si II, Si IV, and C IV) have their resonance transitions in the rest-frame ultraviolet (UV) region. Their observed wavelengths are still in the UV region for low-redshift targets like the MB.

In this paper, we report on the general physical conditions and chemical composition of the MB gas far from the location of stellar populations through quasar absorption line analysis. We chose the sightline toward a radio-loud quasar PKS 0312770 at $z=0.223$ that passes through the MB (Figure 1) at a heliocentric distance of $\sim 50 \mathrm{kpc}$ (similar to the distance of the $\mathrm{SMC}$ ). Because the quasar is radio loud, we are also able to detect neutral hydrogen gas as H I $21 \mathrm{~cm}$ emission/absorption features. Kobulnicky \& Dickey (1999) reported detections of such features with three components at a heliocentric velocity of $V_{\odot} \sim 200 \mathrm{~km} \mathrm{~s}^{-1}$. Based on their fitting results, they estimated a total neutral hydrogen column density of $N_{\mathrm{HI}}=1.2 \times 10^{20} \mathrm{~cm}^{-2}$ and mean spin temperatures of $\left\langle T_{S}\right\rangle=22,29$, and $46 \mathrm{~K}$ for the three components. Although the measured spin temperatures are contaminated by warm gas structures that are not related to the absorbing components, they are similar to the spin temperatures of the LMC $\left(T_{\mathrm{S}}=30-40 \mathrm{~K}\right.$; Mebold et al. 1997; Dickey et al. $1994)$ and the SMC ( $T_{\mathrm{S}}=20-50 \mathrm{~K}$; Dickey et al. 1999), and slightly smaller than that of M31 $\left(T_{\mathrm{S}} \sim 70 \mathrm{~K}\right.$; Braun \& Walterbos 1992).

Recently, Smoker et al. (2005) detected Ca II K lines from the $\mathrm{MB}$ gas in their optical, medium-resolution spectrum of PKS 0312-770. Here we report on detections of 30 additional metal absorption lines in an HST/STIS UV high-resolution spectrum of the same quasar. We report the results of photoionization models in order to probe the physical conditions in the MB along this sightline.

We describe the observations and data reduction in Section 2 and our procedures for line-profile fitting and photoionization modeling with Cloudy (Ferland et al. 1998) in Sections 3 and 4. We present our results in Section 5, and discuss them in Section 6.

\section{OBSERVATIONS AND DATA REDUCTION}

A UV spectrum of the quasar, PKS 0312-770 $\left(m_{V}=\right.$ 16.2), was taken with the Hubble Space Telescope (HST)/ Space Telescope Imaging Spectrograph (STIS) (Kobulnicky 2000), using two echelle gratings: the E140M grating covering $1150 \AA-1700 \AA$ and the E230M grating setting that covers $2130 \AA-2985 \AA$, with $6-10 \mathrm{~km} \mathrm{~s}^{-1}$ velocity resolution. The total exposure times are $37,908 \mathrm{~s}$ for the E140M grating and 6060 $\mathrm{s}$ for the E230M grating, which results in a spectrum with a signal-to-noise ratio $(\mathrm{S} / \mathrm{N})$ of $6-28 \mathrm{pixel}^{-16}(\Delta \lambda \sim 0.015 \AA)$ at $\lambda<1700 \AA$ and $3-8$ pixel $^{-1}(\Delta \lambda \sim 0.04 \AA)$ at $\lambda>2130 \AA$. A journal of the observations is shown in Table 1 .

The data were processed with the standard HST/STIS pipeline, CALSTIS (Brown et al. 2002). Continuum fits were made with standard techniques (Churchill \& Vogt 2001) using the IRAF SFIT task. ${ }^{7}$ The normalized spectrum, after rebinning every $0.15 \AA$ for $\lambda<1700 \AA$ and every $0.40 \AA$ for $\lambda>2130 \AA$, is presented in Figure 2 along with the $1 \sigma$ error spectrum.

The STIS spectrum covers various absorption lines detected at $>5 \sigma$, including $\left(\mathrm{Ly} \alpha, \mathrm{Mg}\right.$ I, $\mathrm{C}_{\mathrm{I}}, \mathrm{O}$ I, $\mathrm{N}$ I, Mg II, Mn II, Fe II, Si II, Ni II, S II, C II, Si III, Si IV, C IV, and Nv) arising either from local absorbers in the Milky Way, from high-velocity clouds (HVC), from the MB or from higher redshift absorbers at $z=0.1983,0.2018,0.2026$, and 0.2029 (Giandoni 2005). Absorption lines arising from the MB (that are our main targets) are marked in Figure 2, and their rest-frame equivalent widths or detection limits are listed in Table 2. We can effectively separate absorption profiles of the MB from those of the Milky Way and the HVC, because their velocity separations are large

\footnotetext{
6 It is typically $\sim 6$ because only a tiny range of wavelength around Ly $\alpha$ emission line has a higher $\mathrm{S} / \mathrm{N}$.

7 IRAF is distributed by the National Optical Astronomy Observatories, which are operated by AURA, Inc., under contract to the National Science Foundation.
} 

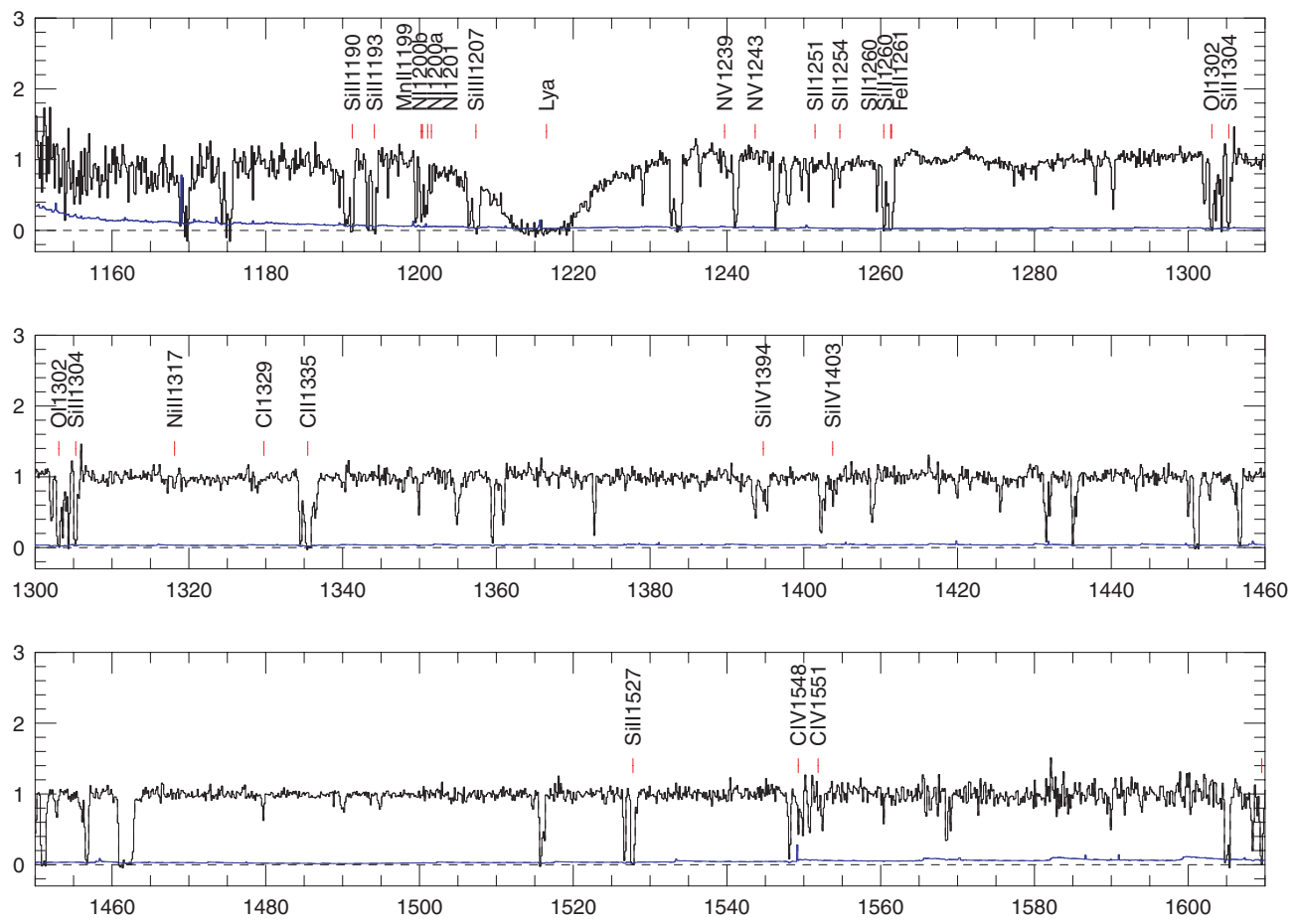

Wavelength $[A]$

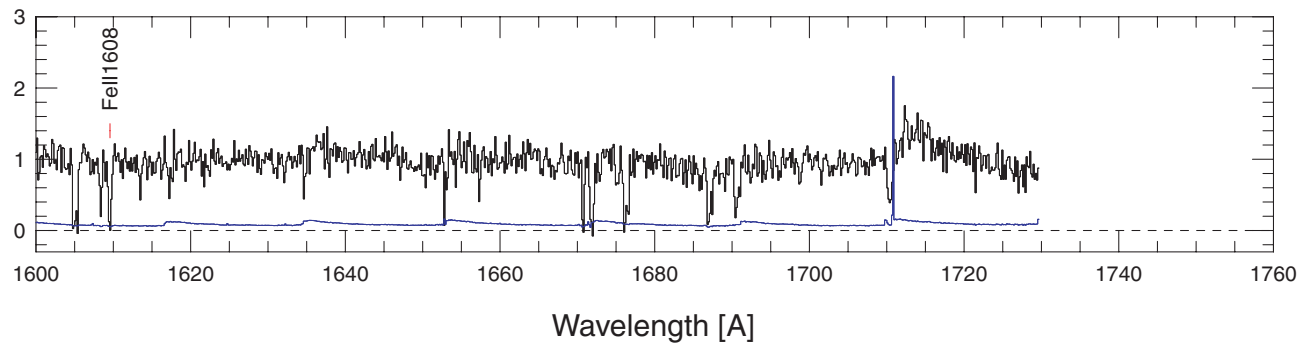

Figure 2. Normalized flux versus wavelength for the HST/STIS E140M and E230M spectra of the quasar PKS 0312-770. The blue histogram displayed beneath the data represents the error spectrum. Positions of absorption lines through the MB at $z \sim 0.0007$ toward the quasar are marked with ticks and transition names. The E140M spectrum at $1150 \AA-1730 \AA$ is binned every $0.15 \AA$, while the E230M spectrum at $2130 \AA-2980 \AA$ is binned every $0.4 \AA$. A Ly $\alpha$ emission around $1216 \AA$ is removed.

(A color version of this figure is available in the online journal.)

enough (the MB and the $\mathrm{HVC}$ are redshifted from the Milky Way by $\sim 200$ and $300 \mathrm{~km} \mathrm{~s}^{-1}$, respectively). Only Ly $\alpha$ is not separated, because it is hidden in the broad damping wing of the Milky Way absorption. The total column density of this system (including contributions from the Milky Way, the MB, and the $\mathrm{HVC}$ altogether) was measured to be $\log N\left(\mathrm{HI} /\left[\mathrm{cm}^{-2}\right]\right) \sim 20.85$ (Giandoni 2005), or 20.87 (Lehner et al. 2008).

As estimated below using photoionization modeling, the total H I column density of the MB toward the quasar PKS $0312-770$ is $\log N\left(\mathrm{HI} /\left[\mathrm{cm}^{-2}\right]\right) \sim 20.2$, just below the lower limit of the criterion for damped Ly $\alpha$ system (DLA). The total rest-frame equivalent width of $\mathrm{Mg}_{\mathrm{II}}\left(W_{r}(2796)=1.91\right.$ $\AA$ ) is also large enough for classification as a strong $\mathrm{Mg}$ II system (whose minimum equivalent width is $W_{r}(2796)=0.3$ $\AA$ A). Absorption systems, classified as strong $\mathrm{Mg}$ II systems, are usually associated with bright $\left(L>0.05 L^{*}\right)$ galaxies within $40 h^{-1} \mathrm{kpc}$ (e.g., Bergeron \& Boissé 1991). The ionization state of the system, in which we detect very strong low-ionization transitions such as $\mathrm{O}_{\mathrm{I}}$ and $\mathrm{Fe}$ II and which has either weak or undetected high-ionization transitions (e.g., $\mathrm{C}$ IV and $\mathrm{Nv}$ ), is very low, as is often the case with DLA systems (e.g.,
Lu et al. 1996). Thus, this system in the MB is probably a local counterpart of DLA systems at higher redshift. We also confirmed that all absorption features in this system are clean without contamination by absorption lines of other systems at higher redshift.

Visual inspection of the velocity plot of the MB system also suggests that the physical and chemical conditions of this system are somewhat similar to those of another absorber detected in the MB toward the young stars, DI 1388 and DGIK 975 (Lehner et al. 2001, 2008; Lehner 2002): (1) having absorption lines with various ionization potentials from $\mathrm{O}_{\mathrm{I}}$ and $\mathrm{C}_{\mathrm{I}}$ to $\mathrm{C}$ IV and $\mathrm{Si}$ IV, and (2) showing strong neutral transitions such as $\mathrm{O}$ I and N I. Lehner et al. (2001) derived several properties of the system toward DI 1388: (1) dust depletion is moderate and its pattern is similar to that of the Galactic halo, and (2) the metallicity is lower than that in the solar neighborhood by $1.1 \mathrm{dex}$, and still lower by 0.5 dex than in the SMC. Using photoionization models, we estimate these parameters toward the quasar, PKS $0312-770$, separated by $\sim 4$. 1 (i.e., $\sim 4.3 \mathrm{kpc}$ at the distance of the SMC) from DI 1388, so that we can study the variety of physical conditions in the MB. 

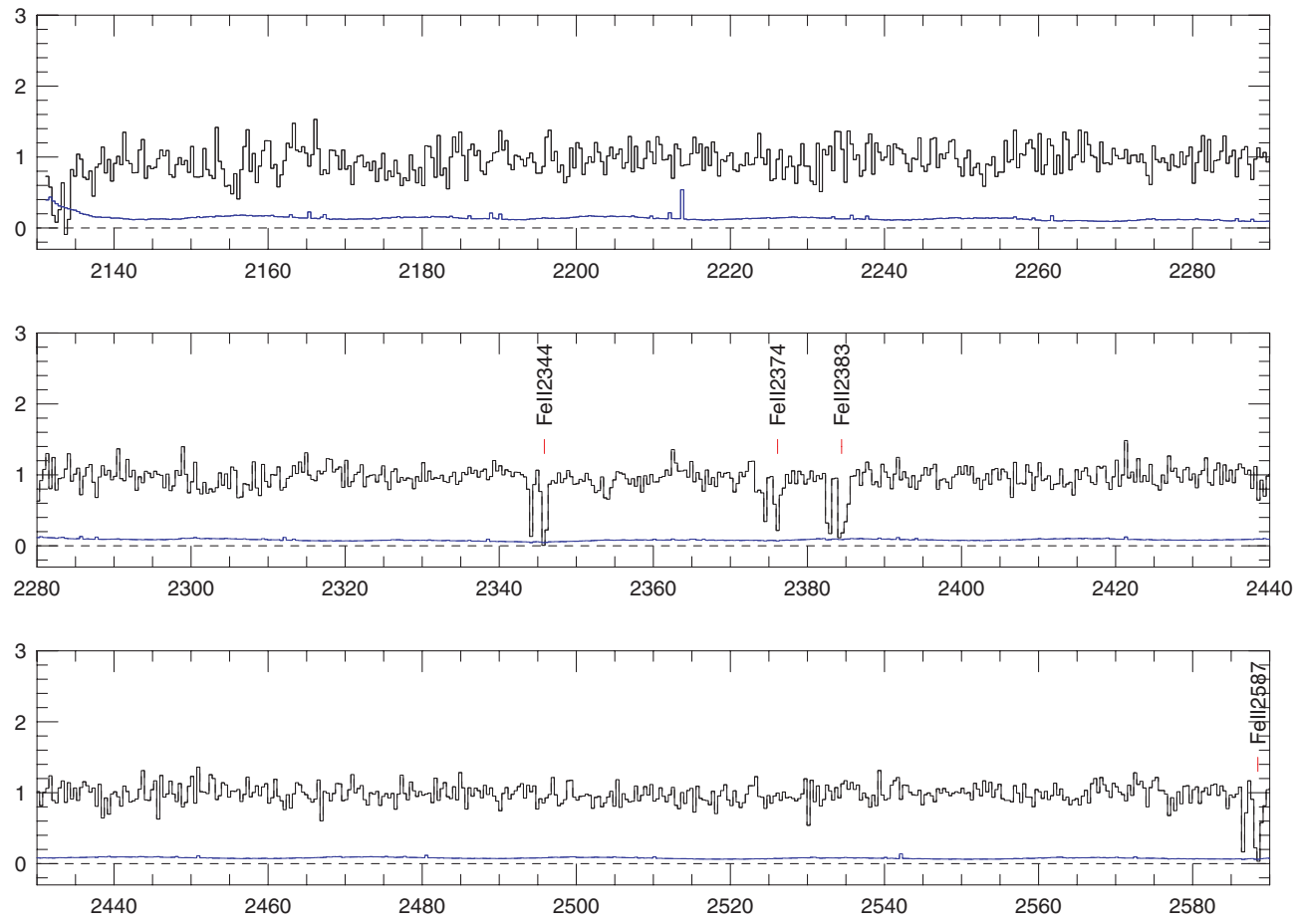

Wavelength $[A]$
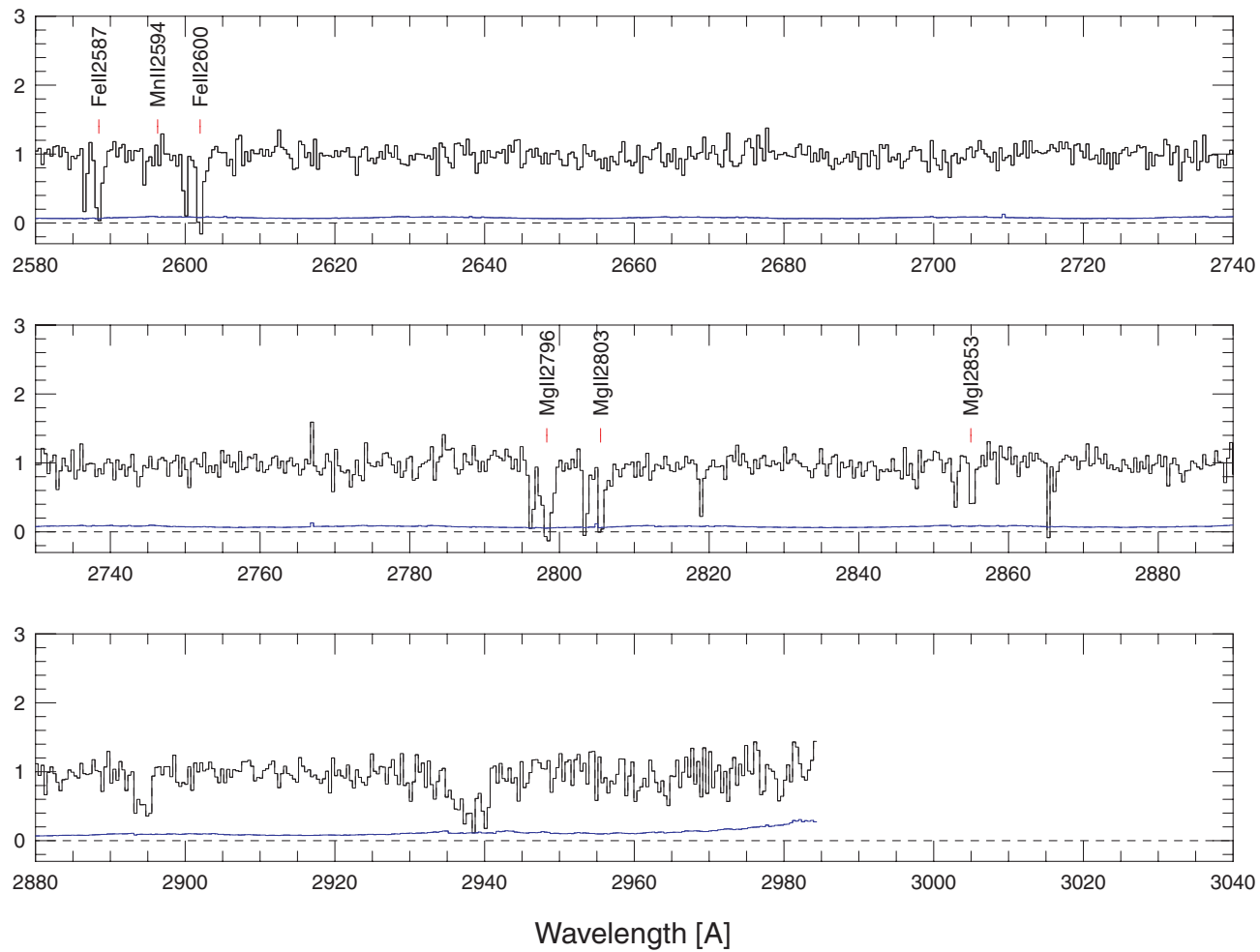

Figure 2. (Continued)

\section{LINE-PROFILE FITTING}

We determine the number of components needed to reproduce the observed spectrum and measure their line parameters (i.e., column density, Doppler parameter, and radial velocity) using Voigt-profile fitting. Kobulnicky \& Dickey (1999) detected three $\mathrm{H}_{\mathrm{I}} 21 \mathrm{~cm}$ absorption components in their radio spectrum of PKS 0312-770 taken with the Australia Telescope Compact Array
(ATCA). The H I $21 \mathrm{~cm}$ absorption lines are due to cool atomic hydrogen regions that probably have similar physical conditions to DLA systems.

Following Kobulnicky \& Dickey (1999), we first used three H I components to fit other metal absorption profiles in our $H S T /$ STIS spectrum. However, we found a three-component fit insufficient, because the line widths of the three components 
Table 2

Observed and Modeled Rest-Frame Equivalent Widths

\begin{tabular}{|c|c|c|}
\hline Transition & $\begin{array}{c}W_{\text {rest }}(\text { spec })^{\mathrm{a}} \\
(\mathrm{m} \AA)^{\mathrm{c}} \\
(2)\end{array}$ & $\begin{array}{c}W_{\text {rest }}(\bmod )^{\mathrm{b}} \\
(\mathrm{m} \AA)^{\mathrm{c}} \\
(3)\end{array}$ \\
\hline $\operatorname{Ly} \alpha$ & $<14.73 \AA^{\mathrm{d}}$ & $8.17 \AA$ \\
\hline $\operatorname{Mg}_{\mathrm{I}} \lambda 2853$ & $496 \pm 51$ & 685 \\
\hline C I $\lambda 1329$ & $18 \pm 4$ & $83^{\mathrm{e}}$ \\
\hline О г $\lambda 1302$ & $520 \pm 7$ & 496 \\
\hline $\mathrm{N}_{\mathrm{I}} \lambda 1200 \mathrm{~b}$ & $<311 \pm 12^{\mathrm{d}}$ & 278 \\
\hline $\mathrm{N}_{\mathrm{I}} \lambda 1200 \mathrm{a}$ & $201 \pm 20$ & 235 \\
\hline $\mathrm{N}_{\mathrm{I}} \lambda 1201$ & $169 \pm 13$ & 179 \\
\hline Mg II $\lambda 2796$ & $1197 \pm 42$ & 1108 \\
\hline $\mathrm{Mg}_{\text {II }} \lambda 2803$ & $904 \pm 43$ & 1063 \\
\hline Mn II $\lambda 1199$ & $<311 \pm 12^{\mathrm{d}}$ & 279 \\
\hline Mn II $\lambda 2594$ & $<49^{f}$ & 134 \\
\hline Fe II $\lambda 1261$ & $<694 \pm 7^{\mathrm{d}}$ & 534 \\
\hline Fe II $\lambda 1608$ & $463 \pm 21$ & 410 \\
\hline Fe II $\lambda 2344$ & $828 \pm 29$ & 765 \\
\hline Fe II $\lambda 2374$ & $539 \pm 42$ & 546 \\
\hline Fe II $\lambda 2383$ & $806 \pm 56$ & 876 \\
\hline Fe II $\lambda 2587$ & $835 \pm 44$ & 780 \\
\hline Fe II $\lambda 2600$ & $1059 \pm 58$ & 938 \\
\hline Si II $\lambda 1190$ & $490 \pm 16$ & 412 \\
\hline Si II $\lambda 1193$ & $495 \pm 14$ & 444 \\
\hline Si II $\lambda 1260$ & $<694 \pm 7^{\mathrm{d}}$ & 533 \\
\hline Si II $\lambda 1304$ & $451 \pm 8$ & 423 \\
\hline Si II $\lambda 1527$ & $563 \pm 9$ & 538 \\
\hline Ni II $\lambda 1317$ & $26 \pm 4$ & 98 \\
\hline S II $\lambda 1251$ & $40 \pm 8$ & 42 \\
\hline$S_{\text {II }} \lambda 1254$ & $120 \pm 7$ & 78 \\
\hline$S_{\text {II }} \lambda 1260$ & $<426 \pm 7^{\mathrm{d}}$ & 110 \\
\hline $\mathrm{C}_{\text {II }} \lambda 1335$ & $<841 \pm 8^{\mathrm{d}}$ & 521 \\
\hline Si III $\lambda 1207$ & $<999 \pm 13^{\mathrm{d}}$ & 300 \\
\hline Si IV $\lambda 1394$ & $78 \pm 7$ & 101 \\
\hline Si IV $\lambda 1403$ & $73 \pm 8$ & 57 \\
\hline C IV $\lambda 1548$ & $94 \pm 12$ & 95 \\
\hline C IV $\lambda 1551$ & $67 \pm 14$ & 51 \\
\hline N v $\lambda 1239$ & $<12^{\mathrm{f}}$ & 2.3 \\
\hline $\mathrm{N} v \lambda 1243$ & $<10^{\mathrm{f}}$ & 1.1 \\
\hline Ca II $\lambda 3935$ & $142 \pm 23^{g}$ & 990 \\
\hline
\end{tabular}

Notes.

${ }^{a}$ Rest-frame equivalent width or detection limit.

${ }^{\mathrm{b}}$ Rest-frame equivalent width of best model (see Section 4).

${ }^{c}$ Except for Ly $\alpha$ for which the unit is Angstroms.

${ }^{\mathrm{d}}$ Blending with other lines.

e This would be $46.8 \mathrm{~m} \AA$ if we assume most likely radiation field, i.e., including extra radiation from the MW and the LMC with $30 \%$ escape fraction (see Section 5).

${ }^{\mathrm{f}}$ Not detected with $5 \sigma$.

${ }^{g}$ Detected in lower resolution spectrum $(R \sim 6000)$ taken with NTT/EMMI by (Smoker et al. 2005).

presented in Kobulnicky \& Dickey (1999; FWHM = 9.6, 13.2, and $10.5 \mathrm{~km} \mathrm{~s}^{-1}$, corresponding to $b(\mathrm{H})=5.8,7.6$, and 6.3 $\mathrm{km} \mathrm{s}^{-1}$ ) could not reproduce the wing profiles at both sides of the metal absorption features. For example, Figure 3 shows a comparison between the observed and modeled $\mathrm{O}_{\mathrm{I}} \lambda 1302$ profiles. We assumed that the Doppler parameters of oxygen and hydrogen were the same for this purpose, i.e., that turbulence dominates $b$, since that yields the maximum value for $b(\mathrm{O})$. If we increase the column density of $\mathrm{O} \mathrm{I}$, damping wings appear before our model reproduces both sides of the observed spectrum. To resolve this discrepancy, we refit the $\mathrm{H}_{\text {I }} 21-\mathrm{cm}$ absorption lines, using the Voigt-profile fitting code (MINFIT; Churchill et al.

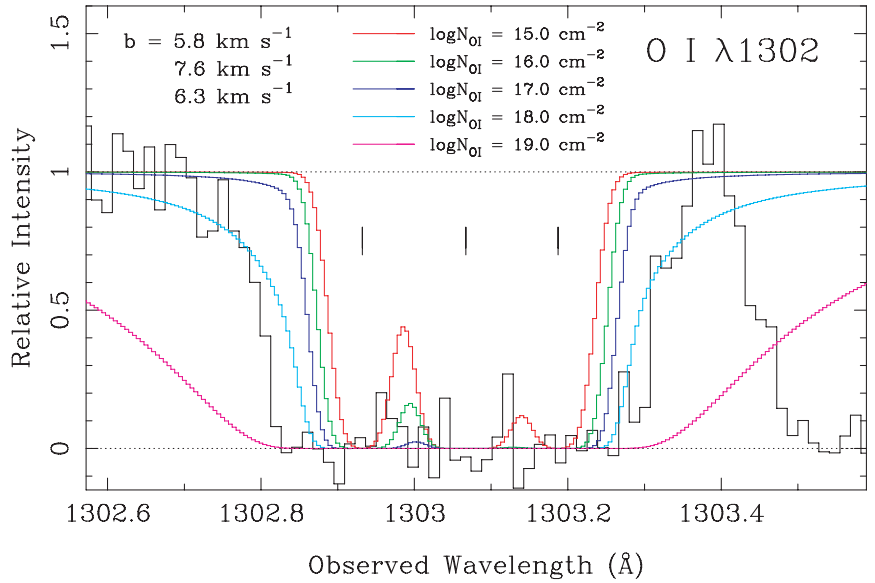

Figure 3. Modeled spectrum with three O I $\lambda 1302$ components whose Doppler parameters are $b=5.8,7.6$, and $6.3 \mathrm{~km} \mathrm{~s}^{-1}$, from left to right (Kobulnicky \& Dickey 1999). Positions of the lines are marked with ticks. Even if we synthesize spectra varying the column density of each component in the range $\log N\left(\mathrm{O} \mathrm{I} /\left[\mathrm{cm}^{-2}\right]\right)=15-19$ (i.e., a total column density of all three components is in the range $\left.\log N\left(\mathrm{OI} /\left[\mathrm{cm}^{-2}\right]\right) \sim 15.5-19.5\right)$, both sides of the observed spectrum shape cannot be reproduced. We test a ridiculously wide range of column density by using same values for three O I components without any justifications, because the purpose of this test is only examine whether the given Doppler parameters could reproduce both sides of the observed absorption profile.

(A color version of this figure is available in the online journal.)

2003; instead of using Gaussian fits as in Kobulnicky \& Dickey 1999).

These measurements of the $\mathrm{H}$ i column densities require some assumptions. We calculated them by

$$
N_{\mathrm{H} \text { I }}=1.823 \times 10^{18}\left(\frac{T_{\mathrm{S}}}{f}\right) \int \tau_{21} d V,
$$

where $T_{\mathrm{S}}$ is the spin temperature in degrees Kelvin, and $\tau_{21}$ is the H ${ }_{\text {I }} 21 \mathrm{~cm}$ optical depth. We adopted the spin temperatures from Kobulnicky \& Dickey (1999). Then by integrating the optical depth of the model profile that we best fit to the observed spectrum, we calculated the H I column densities. Our best-fit parameters are listed in the first three rows of Table 3. The column densities are large enough to be classified as sub-DLAs (i.e., $\left.\left.\log N\left(\mathrm{HI} /\left[\mathrm{cm}^{-2}\right]\right)>19\right)\right)$. The total $\mathrm{H}_{\mathrm{I}}$ column density after adding up the three components will be $N_{\mathrm{H} \mathrm{I}}=1.27 \times 10^{20}$ $\left[\mathrm{cm}^{-2}\right]$, which is consistent with the values measured in past studies using different radio telescopes with different beams on the sky: $(1-5) \times 10^{20}\left[\mathrm{~cm}^{-2}\right]$ (Mathewson \& Ford 1984), (1$2) \times 10^{20}\left[\mathrm{~cm}^{-2}\right]\left(\right.$ Kobulnicky \& Dickey 1999), $1.7 \times 10^{20}\left[\mathrm{~cm}^{-2}\right]$ (Kalberla et al. 2005), $1.3 \times 10^{20}\left[\mathrm{~cm}^{-2}\right]$ (Lehner et al. 2008), and $7.1 \times 10^{20}\left[\mathrm{~cm}^{-2}\right]$ as an upper limit including contributions from our Galaxy (Giandoni 2005). These $\mathrm{H}_{\mathrm{I}}$ line widths are slightly broader than those found previously by Kobulnicky \& Dickey (1999). Using three components with these larger Doppler parameters to fit the $\mathrm{OI}$, we found it possible to reproduce the high-velocity side of $\mathrm{O}_{\mathrm{I}} \lambda 1302$ by adjusting its column density to $\log N\left(\mathrm{O}_{\mathrm{I}} /\left[\mathrm{cm}^{-2}\right]\right) \sim 16$, as shown in Figure $4 .^{8}$

The only remaining disagreement is an underproduction by the model of $\mathrm{O}_{\mathrm{I}} \lambda 1302$ at the low-velocity side of the absorption

\footnotetext{
8 We also cannot reject the alternative solution that instead of a broader $\mathrm{H}_{\mathrm{I}}$ component on the high-velocity side of the system, there are two components blended together at that velocity. Such a model, however, would involve three additional free parameters (i.e., position, column density, and line width) for which we do not have constraints from our radio spectrum. We adopt the one-component model for simplicity.
} 
Table 3

Best-Fit Model for the MB Absorber Toward PKS 0312-770

\begin{tabular}{|c|c|c|c|c|c|c|c|c|c|c|c|c|c|c|}
\hline Transition $^{\mathrm{a}}$ & $\begin{array}{c}V_{\odot}^{\mathrm{b}} \\
\left(\mathrm{km} \mathrm{s}^{-1}\right) \\
(2)\end{array}$ & $\begin{array}{c}\Delta v^{\mathrm{c}} \\
\left(\mathrm{km} \mathrm{s}^{-1}\right) \\
(3)\end{array}$ & $\begin{array}{l}\tau_{\text {cen }}^{\mathrm{d}} \\
(4) \\
\end{array}$ & $\begin{array}{l}T_{\mathrm{S}}^{\mathrm{e}} \\
(\mathrm{K}) \\
(5) \\
\end{array}$ & $\begin{array}{c}\log N^{\mathrm{f}} \\
\left(\mathrm{cm}^{-2}\right) \\
(6) \\
\end{array}$ & $\begin{array}{c}b^{\mathrm{g}} \\
\left(\mathrm{km} \mathrm{s}^{-1}\right) \\
(7)\end{array}$ & $\begin{array}{c}\log \left(\mathrm{Z} / \mathrm{Z}_{\odot}\right)^{\mathrm{h}} \\
(8)\end{array}$ & $\log U^{\mathrm{i}}$ & $\begin{array}{l}T_{\text {gas }}^{\mathrm{j}} \\
(\mathrm{K}) \\
(10) \\
\end{array}$ & $\begin{array}{c}n_{\mathrm{H}}^{\mathrm{k}} \\
\left(\mathrm{cm}^{-3}\right) \\
(11)\end{array}$ & $\begin{array}{c}\text { Size }{ }^{l} \\
(\mathrm{kpc}) \\
(12) \\
\end{array}$ & $\mathrm{N}$ deficiency ${ }^{\mathrm{m}}$ & $\log (\mathrm{Si} / \mathrm{H})$ & $\log (\mathrm{N} / \mathrm{Si})$ \\
\hline $\mathrm{HI}^{n}$ & 175.8 & -34.1 & 0.087 & 22 & 19.59 & 6.4 & -0.7 & -5.7 & 583 & 0.44 & 0.029 & -1.0 & -5.2 & -0.6 \\
\hline $\mathrm{HI}_{\mathrm{I}}$ & 207.3 & -2.6 & 0.049 & 29 & 19.61 & 8.8 & -0.5 & -5.8 & 287 & 0.56 & 0.025 & -0.7 & -5.0 & -0.3 \\
\hline H I & 236.1 & 26.2 & 0.027 & 46 & 19.68 & 11.9 & -0.7 & -5.7 & 501 & 0.44 & 0.035 & -1.4 & -5.2 & -1.0 \\
\hline $\mathrm{Or}^{o}$ & 160.8 & -49.1 & & & (15.00) & $(10.0)$ & -1.0 & -5.1 & 4450 & 0.11 & 0.065 & -0.6 & -5.5 & -0.2 \\
\hline $\operatorname{Si~IV~}^{p}$ & 216.8 & 6.9 & & & 13.16 & 22.1 & -0.6 & -2.6 & 13300 & 0.00035 & 12 & 0.0 & -5.1 & +0.4 \\
\hline
\end{tabular}

Notes.

a Name of transition that is optimized for the Cloudy model.

${ }^{\mathrm{b}}$ Heliocentric velocity.

${ }^{c}$ Relative velocity from the system center whose heliocentric velocity is $209.9 \mathrm{~km} \mathrm{~s}^{-1}$.

d Absorption optical depth at the line center of $\mathrm{H} \mathrm{I} 21 \mathrm{~cm}$, if Column 1 is $\mathrm{H} \mathrm{I}$.

e Spin temperature measured from H I $21 \mathrm{~cm}$ emission line, if Column 1 is $\mathrm{H} \mathrm{I}$.

${ }^{\mathrm{f}}$ Best-Voigt-profile fit value of column density of the optimized line in Column 1.

g Best Voigt-profile fit value of Doppler parameter of the optimized line in Column 1.

${ }^{\mathrm{h}}$ Best-model parameter of metallicity.

i Best-model parameter of ionization parameter.

$\mathrm{j}$ Gas temperature from the best model.

k Total hydrogen volume density per cubic centimeter. This depends very strongly on the radiation field's absolute strength (see Section 5.5).

${ }^{1}$ Thickness of the absorber assuming a plane-parallel structure. This depends very strongly on the radiation field's absolute strength (see Section 5.5).

m Nitrogen Deficiency, compared to the Solar abundance pattern.

${ }^{\mathrm{n}}$ We adopt the same parameters as for the $236 \mathrm{~km} \mathrm{~s}^{-1}$ cloud, as an example of acceptable model.

${ }^{\mathrm{o}}$ This is an example of the best-fit models that have acceptable range of parameters, $\log \left(Z / Z_{\odot}\right)>-1.0$ and $\log U>-6.0$.

$\mathrm{p}$ We adopt the typical metallicity of the SMC, as an example of acceptable models.

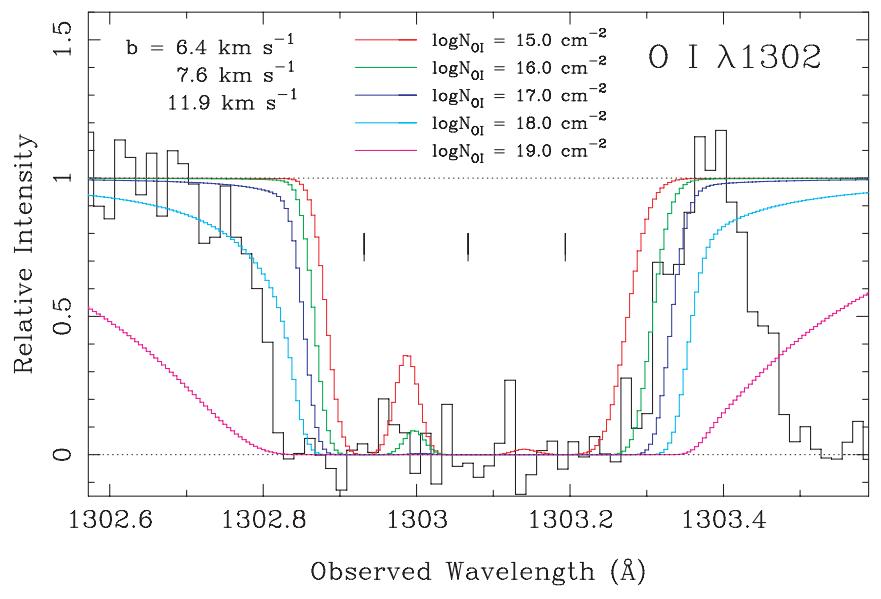

Figure 4. Same as Figure 3, but the three O I $\lambda 1302$ components have Doppler parameters of $b=6.4,7.6$, and $11.9 \mathrm{~km} \mathrm{~s}^{-1}$ from our own fitting trials. A model with $\log N\left(\mathrm{O} \mathrm{I} /\left[\mathrm{cm}^{-2}\right]\right)$ of 16.0 gives an acceptable fit at the high-velocity side of the observed spectrum.

(A color version of this figure is available in the online journal.)

profile. Because a similar discrepancy is seen in other metal absorption lines, such as Mg II, Fe II, and Si II, there is probably an additional component at the low-velocity side of this system. Assuming a fiducial line width of $b=10 \mathrm{~km} \mathrm{~s}^{-1}$ (i.e., the average Doppler parameter of the other three H I $21-\mathrm{cm}$ lines is $9.0 \mathrm{~km} \mathrm{~s}^{-1}$ ), we adjust both the line position and column density of $\mathrm{O}_{\mathrm{I}}$ and find the best combination: $V_{\odot}=160.8 \mathrm{~km} \mathrm{~s}^{-1}$ and $\log N\left(\mathrm{O} \mathrm{I} /\left[\mathrm{cm}^{-2}\right]\right) \sim 15$.

In fact, another strong constraint for this additional component comes from the radio observation. We can place an upper limit on the H $21 \mathrm{~cm}$ line optical depth of this additional component at the line center, because we did not detect it at the appropriate wavelength in the $\mathrm{HI} 21 \mathrm{~cm}$ absorption spectrum. The $\mathrm{S} / \mathrm{N}$ of the spectrum is $\sim 40$ at that wavelength, which corre- sponds to a $1 \sigma$ optical depth limit of $\tau_{\max }=-\ln (1-\sigma) \sim 0.025 .{ }^{9}$ As described in the following section, our best photoionization model for this component gives $\log N\left(\mathrm{HI} /\left[\mathrm{cm}^{-2}\right]\right)$ of 19.31 and $b$ of $13.0 \mathrm{~km} \mathrm{~s}^{-1}$ for the $\mathrm{H}$ I line, whose optical depth at line center would be $\tau=0.022$, smaller than the $1 \sigma$ detection limit in the radio spectrum (Figure 5). We assume the same spin temperature for this component as was measured for the closest component at $V_{\odot}=175.8 \mathrm{~km} \mathrm{~s}^{-1}\left(T_{\mathrm{S}}=22 \mathrm{~K}\right)$. The line parameters for this additional component, determined from a fit to the $\mathrm{O}$ I are listed in the fourth line of Table 3. However, we will not consider this component in the discussion (Section 6), because the fitted line parameters are all based on an arbitrary Doppler parameter.

\section{PHOTOIONIZATION MODELING}

\subsection{Modeling Procedure}

We briefly summarize our modeling procedure, although it is similar to previous studies (e.g., Churchill \& Charlton 1999). Using the photoionization code Cloudy, version 07.02.00 (Ferland et al. 1998), for each of the five clouds (the fifth one is introduced later) we search for the best combinations of the fit parameters: (1) ionization parameter $\left(\log U=\log \left[n_{\gamma} / n_{\mathrm{H}}\right]\right.$, defined as the ratio of ionizing photons to the number density of hydrogen in the absorbing gas) and (2) metallicity $\left(\log \left(Z / Z_{\odot}\right)\right)$ in solar units. ${ }^{10}$ The MB is likely to be confined and compressed by its interaction with the Galactic halo, but this does not produce shock ionization (Bland-Hawthorn et al. 2007). Therefore, we consider only photoionization. We optimize on the observed column densities of $\mathrm{H}$ I or other metal ions (i.e., O I and Si IV)

\footnotetext{
9 That is, a typical uncertainty of our estimates of $\mathrm{HI}$ column densities is also about $2.5 \%$.

${ }^{10}$ The default solar composition we assume is listed in Hazy 1, a manual of Cloudy. For example, $[\mathrm{Si} / \mathrm{H}]_{\odot},[\mathrm{N} / \mathrm{H}]_{\odot}$, and $[\mathrm{O} / \mathrm{H}]_{\odot}$ are $-4.46,-4.07$, and -3.31 , respectively.
} 


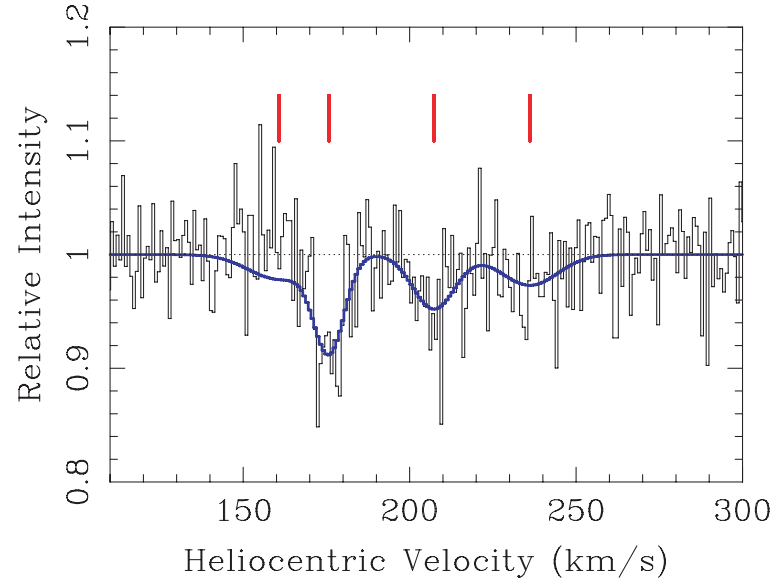

Figure 5. Modeled spectrum of the $\mathrm{H}_{\mathrm{I}} 21 \mathrm{~cm}$ absorption lines, overlaid with the radio spectrum of PKS 0312-770 taken with the Australia Telescope Compact Array (Kobulnicky \& Dickey 1999). In addition to the original three components (Kobulnicky \& Dickey 1999), we add another component at $\Delta v=-49 \mathrm{~km} \mathrm{~s}^{-1}$ (i.e., $V_{\odot}=160.8 \mathrm{~km} \mathrm{~s}^{-1}$ ), required by the modeling of the metal lines. This additional component (whose central optical depth is $\tau=0.022$ ) is not detected at more than a $1 \sigma$ level in the observed spectrum whose $\mathrm{S} / \mathrm{N}$ is about $40 \mathrm{pixel}^{-1}$ (which corresponds to the detection limit of $\tau=0.025$ at the line center).

(A color version of this figure is available in the online journal.)

that are listed in the first column of Table 3, that is we require the Cloudy models to produce those column densities. For example, in the case of the component at $V_{\odot} \sim 176 \mathrm{~km} \mathrm{~s}^{-1}$, we always fix the $\mathrm{H}$ i column density to $\log N\left(\mathrm{H} \mathrm{I} /\left[\mathrm{cm}^{-2}\right]\right)=19.59$, and search for the best values of $\log U$ and $\log \left(Z / Z_{\odot}\right)$ in a grid with steps of $0.1 \mathrm{dex}$, to reproduce as many absorption lines from other transitions as possible. We repeat this procedure for the other four components, individually. We assume that the absorbers are plane-parallel structures of constant density, and that they are in photoionization equilibrium. We initially adopt a solar abundance pattern, but also explore some variations based on the observational constraints. For the incident radiation field, we first consider a pure extragalactic background radiation with contributions from quasars and star-forming galaxies (with a photon escape fraction of 0.1), following Haardt \& Madau (1996, 2001). We also explore the effects of other incident radiation fields, including fluxes from our Galaxy and the LMC, as described in the following section.

For a given $\log U$ and $\log \left(Z / Z_{\odot}\right)$, Cloudy computes the column density of each element in various ionization stages, and the equilibrium gas temperature, $T$. We can calculate the Doppler parameter for each element using $b=\sqrt{b_{\mathrm{T}}^{2}+b_{\mathrm{turb}}^{2}}$, where $b_{\mathrm{T}}$ is the thermal broadening defined as $b_{\mathrm{T}}=\sqrt{2 k T / m}$, and $b_{\text {turb }}$ is the broadening from gas turbulence and bulk motion. We use the measured $b$ for the transition on which we optimized (listed in Table 3) in order to calculate $b_{\text {turb }}$, which is then applied for other elements. Using these derived line parameters from the model, we synthesize a spectrum, after convolving the instrumental line spread function of HST/STIS, and compare it to the observed spectrum. The synthesized spectra are compared to the observed spectrum by eye, because models with metallicity and ionization parameters that differ only slightly from the best values (e.g., 0.1 or 0.2 dex) would deviate significantly from the observed spectrum (see Figure 4 of Misawa et al. 2008). Moreover, a formal procedure like $\chi^{2}$ fitting is not applicable, because the system in the MB has various (more than 10) transitions that we must consider simultaneously, and it is very difficult to determine how they should be weighted in a $\chi^{2}$ calculation (e.g., Misawa et al. 2008).

The transitions we optimize are $\mathrm{HI}$ and $\mathrm{O} \mathrm{I}$, and both have very low ionization potentials (IP $=13.6 \mathrm{eV}$ ). As frequently reported for photoionization models of $\mathrm{Mg}$ II absorbers, the lowionization phase clouds that produce $\mathrm{Mg}$ II absorption lines also produce other low-ionization transitions (e.g., C II, Si II, and Fe II), but not high-ionization transitions (e.g., Si IV and C IV). An additional high-ionization phase is almost always required to reproduce these transitions. Therefore, we repeat line fitting and photoionization modeling for the high-ionization phase by optimizing high-ionization transitions (Si IV in this study, as described below). Finally, we synthesize a model spectrum including both low- and high-ionization phases, and compare it to the observed spectrum.

As described above, our method for deriving constraints on metallicities and ionization parameters of the absorbing gas relies on Voigt-profile fitting of separate components of the absorption profile of a particular transition which we feel is best constrained. We then use photoionization modeling to infer the column densities and Doppler parameters of other transitions in these Voigt-profile component clouds. Synthesized profiles from these models are compared to the data in order that we can place constraints on the parameters. Although this method does rely on the assumptions behind the Cloudy models, it has some advantages over simply taking the ratios of apparent column densities of selected transitions in order to determine metallicity. First of all, we can separately examine properties of individual clouds along the line of sight at different velocities. We are not averaging these components together, which the apparent column density method requires, since it does not distinguish, for example, how much of the hydrogen is associated with the separate clouds. Second, we can use the components determined from unsaturated lines in order to constrain the properties of those clouds using other saturated or partially saturated components as well. The measured Doppler parameters for the unsaturated lines can be used along with the temperature given by a given Cloudy model in order to determine model Doppler parameters of other lines that may be saturated or blended. Comparing the model to the shapes of the observed profiles of these lines, particularly the shapes of the sides of the profiles, often yields meaningful constraints on parameters. Models also take into account the appropriate ionization corrections in each case. In this way, we can determine the range of acceptable parameters, considering all observational constraints. Finally, we are able to consider separate phases of gas, having different densities and velocities along the line of sight, by comparing model predictions to the observations, component by component.

\subsection{Alternate Incident Radiation Fields}

Because the Milky Way (MW) and the LMC are located within several tens of kiloparsec of the MB, they also contribute as additional ionizing radiation sources. Therefore, we consider three alternative incident radiation fields, (1) extra-galactic background (EGB; Haardt \& Madau 1996, 2001) plus radiation from the $\mathrm{MW}$ at a distance of $D=50 \mathrm{kpc}$ from the MW (Fox et al. 2005), (2) a maximum flux model, with the EGB, the MW radiation, plus radiation from the LMC with a $30 \%$ escape fraction, and (3) an intermediate case, with EGB, the MW radiation, plus radiation from the LMC with a $15 \%$ 


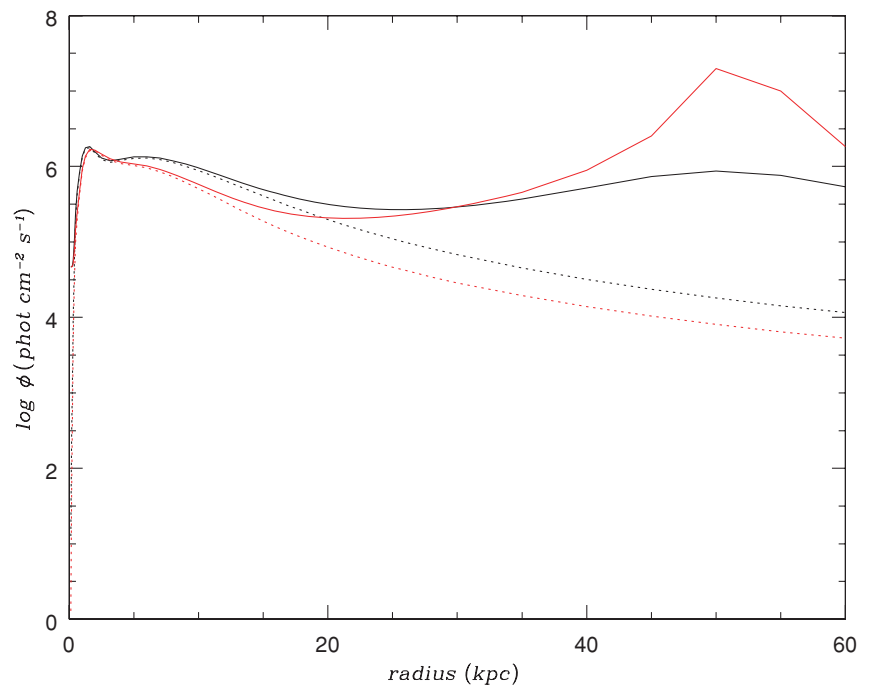

Figure 6. Strength of the ionizing radiation field as a function of distance along our sightline toward PKS 0132-770. The solid curve includes the radiation from the LMC as well as the radiation from the MW, while the dotted curve includes only the latter. The black curves are for the direction to PKS 0312-770, while the red curves are for the direction straight toward the LMC. For example, at a radius of $50 \mathrm{kpc}$, the ionization strength decreases from (1) $\mathrm{LMC}+\mathrm{MW}$ toward LMC, (2) LMC + MW toward PKS 0312-770, (3) MW toward PKS 0312-770, to (4) MW toward LMC. The LMC model assumes that $30 \%$ of the ionizing radiation escapes from the LMC, which we consider to be an upper limit.

(A color version of this figure is available in the online journal.)

escape fraction. ${ }^{11}$ We construct these radiation fields following the procedure of Bland-Hawthorn \& Maloney (1999, 2002). Figure 6 shows the strength of the ionizing radiation from the MW and the LMC as a function of a distance from the center of the MW. At $D \sim 50 \mathrm{kpc}$, at which the MB is located, the contribution from the LMC to the radiation field is significant. On the other hand, the contribution from the SMC is negligible because of its low luminosity.

The spectral shapes for each of the four incident radiation field models on the MB gas are plotted in Figure 7. The radiation from the MW and the LMC start to dominate the EBR at $\log (v /[\mathrm{Hz}])$ $<16.1$, and strongly dominate over the EBR at $\log (v /[\mathrm{Hz}])<$ 15.5. Normalizations for these fields, in units of the density of ionizing photons, and the contribution from the local flux sources (MW and LMC) compared to the EBR are summarized in Table 4.

\section{RESULTS}

Transitions that are detected in the HST/STIS spectrum and those that provide useful limits are shown in Figure 8 , from the lowest $(\mathrm{Mg}$ I, IP $=7.6 \mathrm{eV})$ to highest $(\mathrm{Nv}, \mathrm{IP}=97.9$ $\mathrm{eV}$ ) ionization potentials, following the $\mathrm{Ly} \alpha$ profile in the first panel. We also display the observed $\mathrm{H}_{\mathrm{I}} 21 \mathrm{~cm}$ absorption profile (Kobulnicky \& Dickey 1999). In Figure 8, $0 \mathrm{~km} \mathrm{~s}^{-1}$ denotes the apparent optical depth-weighted median of the MB absorption system, corresponding to a heliocentric velocity of $V_{\odot}=209.9$ $\mathrm{km} \mathrm{s}^{-1}$. A number of absorption lines from various ions (i.e., Mg I, C I, O I, N I, Mg II, Mn II, Fe II, Si II, Ni II, S II, Si IV, and

\footnotetext{
11 We do not consider the contribution from local O/B-type stars in the MB, because it is less likely that our sightline to the background quasar goes through stellar associations, at least compared to the sightlines toward stars in the MB. It is also suggested that O/B-type stars tend to localize in the wing of the SMC (Irwin et al. 1990; Battinelli \& Demers 1992).
}

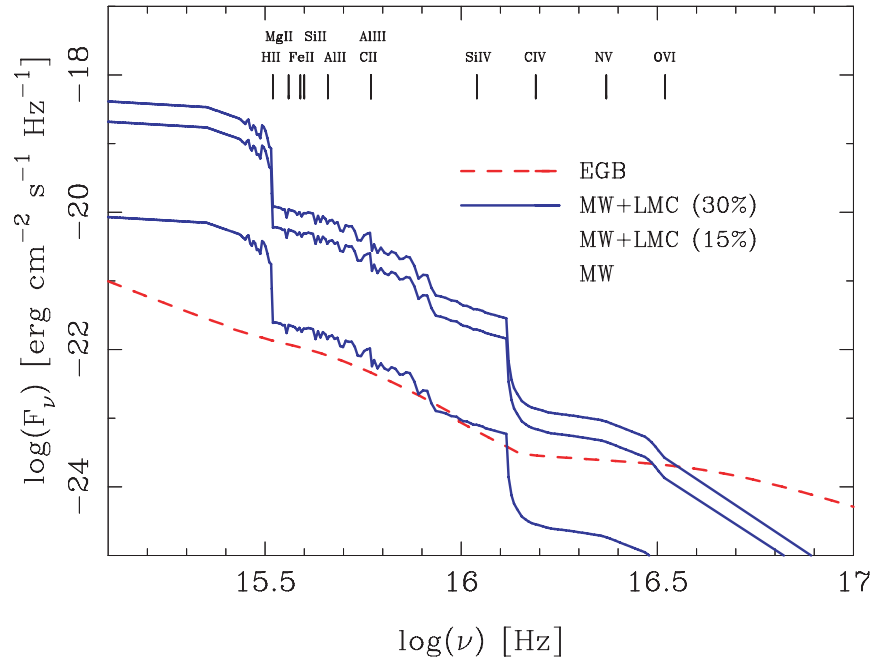

Figure 7. Spectrum of four different radiation fields that we applied in our photoionization calculations with Cloudy. The smooth, dashed, red line denotes the extragalactic background radiation (EBR) from Haardt \& Madau (1996, 2001). The three solid blue lines represent the radiation fields from the MW, $\mathrm{MW}+\mathrm{LMC}$ with $15 \%$ escape fraction, and MW $+30 \%$ escape fraction, from bottom to top. These are normalized assuming a distance of $D=50 \mathrm{kpc}$ from the center of the MW to the PKS 0312-770 sightline cloud. Ionization edges of several important transitions are indicated at the top of the plot.

(A color version of this figure is available in the online journal.)

Table 4

Summary of Incident Radiation Models

\begin{tabular}{lcccccc}
\hline \hline Model & EBR & MW & LMC & $\begin{array}{c}\log n_{\gamma}(\mathrm{EBR})^{\mathrm{a}} \\
\left(\mathrm{cm}^{-3}\right)\end{array}$ & $\begin{array}{c}\log n_{\gamma}(\mathrm{MW}+\mathrm{LMC})^{\mathrm{b}} \\
\left(\mathrm{cm}^{-3}\right)\end{array}$ & $f^{\mathrm{c}}$ \\
\hline 1$)$ & $(2)$ & $(3)$ & $(4)$ & $(5)$ & $(6)$ & $(7)$ \\
\hline 1 & Y & & & -6.06 & $\ldots$ & $\ldots$ \\
2 & Y & Y & & -6.06 & -6.22 & 0.69 \\
3 & Y & Y & $15 \%$ & -6.06 & -4.84 & 16.6 \\
4 & Y & Y & $30 \%$ & -6.06 & -4.54 & 33.1 \\
\hline
\end{tabular}

Notes.

a Volume density of hydrogen-ionizing photons from the extragalactic background radiation (Haardt \& Madau 1996, 2001).

b Volume density of hydrogen-ionizing photons from the MW and the LMC (Fox et al. 2005).

${ }^{c}$ Ratio of the ionizing photon number densities from the MW and/or the LMC, to that from the EBR, $n_{\gamma}(\mathrm{MW}+\mathrm{LMC}) / n_{\gamma}(\mathrm{EBR})$.

$\mathrm{C}$ IV) are detected. For $\mathrm{Nv}$, we can place only an upper limit on the equivalent width. The $\mathrm{Mg}$ II $\lambda 2803$ profile suffers from a data defect on its blue side, so we use the $\mathrm{Mg}$ II $\lambda 2796$ profile as the main $\mathrm{Mg}$ II constraint. Since the $\mathrm{Mg}$ I $\lambda 2853$ profile is noisy (i.e., $\mathrm{S} / \mathrm{N} \sim 3.7$ pixel $^{-1}$ ), it is only used as a loose constraint on models.

As a starting point for photoionization modeling, we roughly estimate possible ranges of gas temperature and ionization parameter $(\log U)$. The observed line widths of all transitions are similar, which suggests that bulk motion (gas turbulence) is a dominant source of line broadening. The relatively narrow $\mathrm{H} \mathrm{I}$ $21 \mathrm{~cm}$ lines, especially the one with $b=6.4 \mathrm{~km} \mathrm{~s}^{-1}$, then imply that the gas temperature is very low. Such a low temperature implies that the ionization parameter is also very small $(\log U$ $\leqslant-5.0)$. For the metallicity, the value in the SMC has been estimated as $Z=0.1-0.2 Z_{\odot}$ (Pagel et al. 1978; Welty et al. 2001). Therefore, in the following, we explore metallicities and ionization parameters, in steps of $0.1 \mathrm{dex}$, from $\log U=-7.0$ to -4.0 and $\log \left(Z / Z_{\odot}\right)=-1.0$ to 0.0 unless other parameter ranges are suggested. 

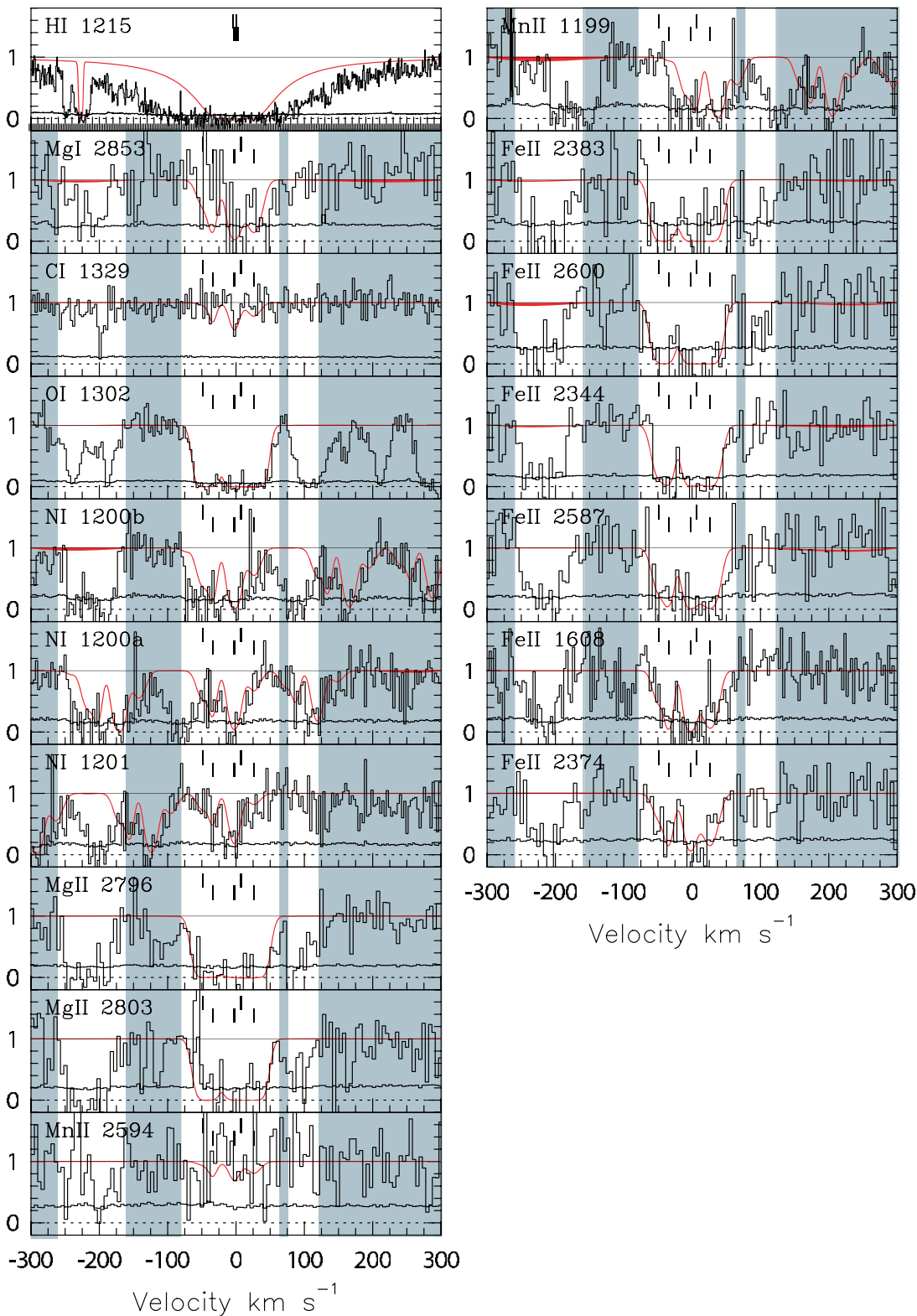

Figure 8. Detected transitions, and those that provide limiting constraints, shown in velocity space for the absorption system in the Magellanic Bridge toward the

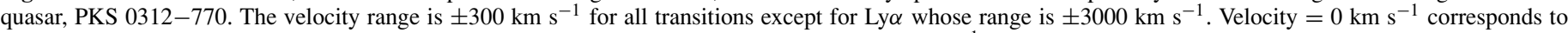
the system center of the Magellanic Bridge absorber whose heliocentric velocity is $V_{\odot}=209.9 \mathrm{~km} \mathrm{~s}^{-1}$. The data are from a HST/STIS spectrum. The error spectrum is also indicated as a solid histogram just above the dotted line crossing each plot at zero flux. An example of the best model fit to the observed spectrum (using model parameters listed in Table 3) is superimposed on the data as a solid (red) curve. Three unshaded regions denote the locations of the Milky Way, the MB, and a high-velocity cloud (from left to right), respectively. The positions of absorption components are marked with ticks. Above the spectrum in each panel, three ticks in the bottom line are H I optimized components, while those in the top line are O I or Si IV optimized components.

(A color version of this figure is available in the online journal.)

\subsection{Three H $21 \mathrm{~cm}$ Clouds}

At first, we seek the best photoionization model parameters for the three H $21 \mathrm{~cm}$ clouds at $\Delta v=-34,-3$, and $26 \mathrm{~km}$ $\mathrm{s}^{-1}$ from the system center (i.e., $V_{\odot}=176,207$, and $236 \mathrm{~km}$ $\mathrm{s}^{-1}$ ) that we determined in Section 3 by optimizing a fit to the H I $21 \mathrm{~cm}$ absorption line. We require our model to reproduce the column density of $\mathrm{H}_{\mathrm{I}}$ from that fit. Because the cloud at $\Delta v=-3 \mathrm{~km} \mathrm{~s}^{-1}$ has clear detections in $\mathrm{C}$ I, N I, Ni II, and $\mathrm{S}$ II, without blending with other absorption features, we begin with this cloud. We can constrain the metallicity to be $-0.9 \leqslant$ $\log \left(Z / Z_{\odot}\right) \leqslant-0.5 /-0.5 \leqslant \log \left(Z / Z_{\odot}\right) \leqslant-0.2$ to avoid over $/$ underproduction of $\mathrm{Ni}$ II and $\mathrm{S}$ II. Therefore, the acceptable metallicity is $\log \left(Z / Z_{\odot}\right) \sim-0.5$. With this metallicity, the ionization parameter must be $-6.0<\log U<-4.8$ to avoid over/underproduction of $\mathrm{C}$ I. Even our favored model, with $\log \left(Z / Z_{\odot}\right) \sim-0.5$ and $\log U \sim-5.8$, for this $\Delta v=-3 \mathrm{~km} \mathrm{~s}^{-1}$ cloud substantially overproduces the $\mathrm{N}_{\mathrm{I}} \lambda 1201$ absorption. To reconcile the model with the observed $\mathrm{N}_{\mathrm{I}} \lambda 1201$ absorption, we decrease the nitrogen abundance by a factor of 0.7 dex compared to its solar abundance pattern. The production mechanisms of nitrogen are poorly understood. However, a nitrogen deficiency in the SMC has already been reported (e.g., Mallouris et al. 2001), and similar deficiencies are seen in some DLA systems 
MB System toward PKS 0312-770
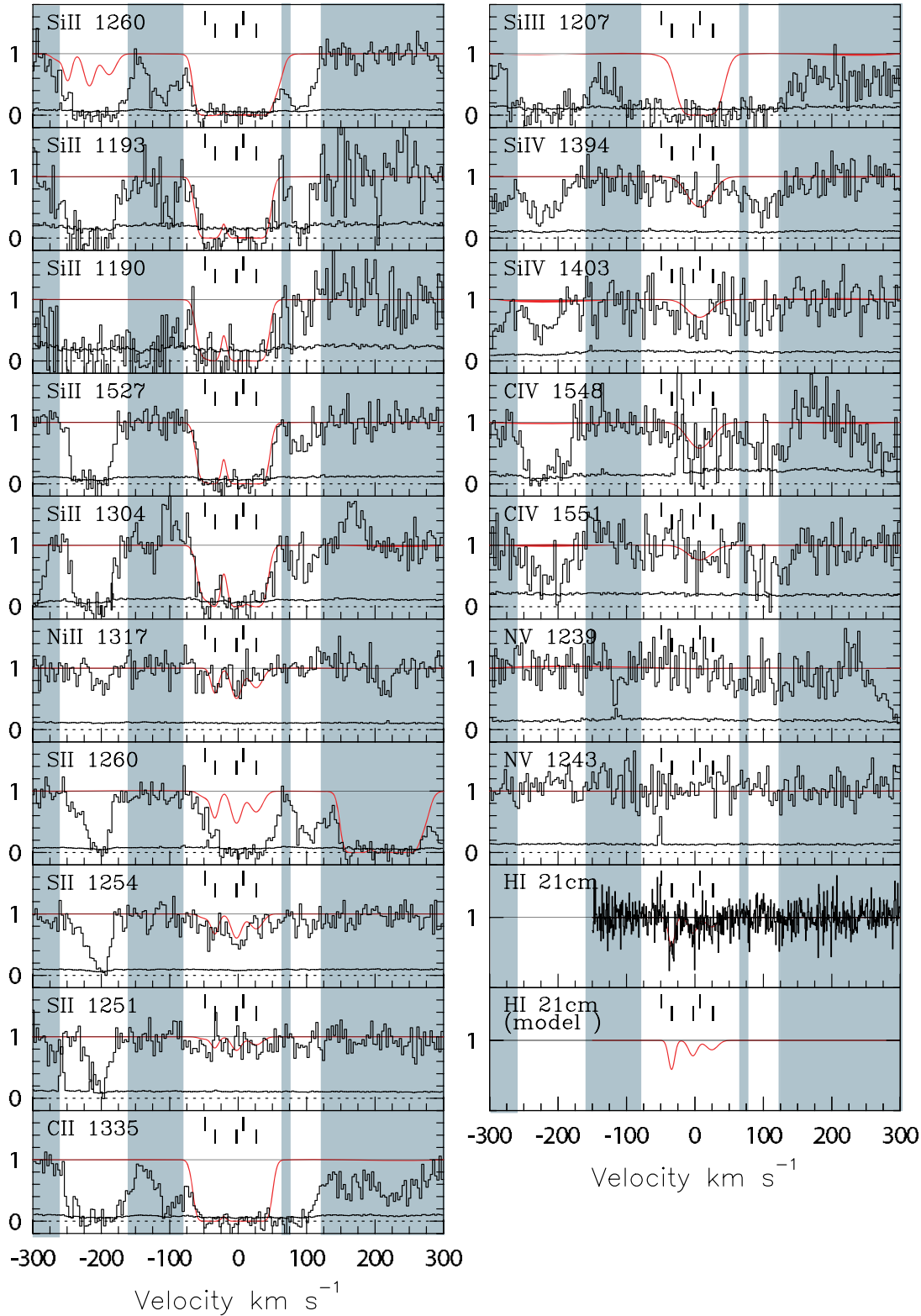

Figure 8. (Continued)

(e.g., Pettini et al. 2002) and weak Mg II systems (Zonak et al. 2004). Since O I and H I are strongly coupled in terms of their ionization, their ratio is often used as a measure of metallicity (Lehner et al. 2008). In our case, the $\mathrm{O}$ I is highly saturated so that the Voigt-profile components cannot be measured directly from the profile. However, once we have determined the metallicity from the weaker $\mathrm{Ni}$ II and $\mathrm{S}$ II profiles, we can verify that the favored model produces an $\mathrm{O}$ I/H $\mathrm{H}_{\text {I }}$ ratio consistent with these metallicities. For the $-3 \mathrm{~km} \mathrm{~s}^{-1}$ component, we find $\log \left[\mathrm{N}\left(\mathrm{O}_{\mathrm{I}}\right) / \mathrm{N}\left(\mathrm{HI}_{\mathrm{I}}\right)\right]=-3.81$, corresponding to $\log \left(Z / Z_{\odot}\right)=$ -0.5 . The fact that this is consistent with the metallicity inferred from the weaker profiles confirms that ionization corrections are inferred properly from our models, and that the saturated $\mathrm{O}_{\mathrm{I}}$ profile has been separated self-consistently into Voigt-profile components

Next, we consider the $\mathrm{H}$ I cloud at $\Delta v=26 \mathrm{~km} \mathrm{~s}^{-1}$. Because this component has the largest recessional velocity, it should reproduce at the high-velocity side of all absorption features seen in the $\mathrm{OI}, \mathrm{Mg}$ II, Fe II, and Si II lines. We also require that the component reproduces the weak Ni II absorption. The Si II provides the best lower limit on metallicity, $\log \left(Z / Z_{\odot}\right)$ $>-0.8$, because the less saturated Si II $\lambda 1304$ profile is available. Similarly, an upper $\operatorname{limit}$ of $\log \left(Z / Z_{\odot}\right)<-0.6$ applies in order that $\mathrm{Ni}$ II is not overproduced. At the preferred value of $\log \left(Z / Z_{\odot}\right) \sim-0.7$, there is a strict lower limit on the ionization parameter of $\log U>-6.1$ under which $\mathrm{C}_{\text {I }}$ would be overproduced. We do not place any formal upper limit on the ionization parameter of this cloud, since the constraints from the relatively low signal-to-noise spectrum were not significant. However, we can place a marginal upper limit, $\log U<-5.0$ to avoid an underproduction of Si II, once we adopt $\log \left(Z / Z_{\odot}\right)=$ -0.7 . We favor values toward the lower end of this range, $\log U$ $\sim-6.0$, because the fits to $\mathrm{OI}_{\mathrm{I}}, \mathrm{Mg}$ II, and Fe II are slightly better. We also find that this cloud must have a deficiency of 
nitrogen of 1.4 dex compared to the solar value, although this value could be smaller (Section 6).

Because the third H I cloud at $\Delta v=-34 \mathrm{~km} \mathrm{~s}^{-1}$ is heavily blended with the adjacent clouds, it is difficult to place strict constraints on its physical conditions. Therefore, we simply note that its metallicity and ionization parameter could be very close to those of the cloud at $\Delta v=26 \mathrm{~km} \mathrm{~s}^{-1}$. Several transitions, Mg I, Ni II, and CI, are overproduced for metallicities higher than $\log \left(Z / Z_{\odot}\right)>-0.6$ or -0.7 . The metallicity should also be greater than -1.0 , to avoid an underproduction of Si II. Although we cannot place strong constraints on the ionization parameter, the $\log U$ should be between -6.0 and -5.0 in the acceptable range of the metallicity, $-1.0<\log \left(Z / Z_{\odot}\right)<-0.7$, to reproduce the observed profiles of $\mathrm{C}$ I, Si II, and O I. We adopt a model with $\log U \sim-5.7$ and $\log \left(Z / Z_{\odot}\right) \sim-0.7$, the same as for the $\Delta v=26 \mathrm{~km} \mathrm{~s}^{-1}$ cloud, because it is consistent with the observations. We also find that this cloud must have a deficiency of nitrogen of 1.0 dex compared to the solar value.

\subsection{High-Ionization Cloud}

In addition to the three $\mathrm{H}$ I clouds above, one high-ionization cloud, at $\Delta v=7 \mathrm{~km} \mathrm{~s}^{-1}$ (i.e., $V_{\odot}=217 \mathrm{~km} \mathrm{~s}^{-1}$ ), is necessary to reproduce absorption in the high-ionization lines, Si IV and $\mathrm{C}$ IV. To determine the photoionization model parameters for this cloud, we optimize on the Si IV column density, since the Voigt-profile fitting is better in this region than it is for the C IV doublet. The result of a Voigt-profile fit to the Si IV is given in the last row of Table 3. Comparing to the observed C IV absorption, we find an ionization parameter of $-2.7 \leqslant$ $\log U \leqslant-2.4$. The metallicity of the high-ionization cloud is constrained to be $\log \left(Z / Z_{\odot}\right)>-4.0$ by the requirement that the corresponding $\mathrm{HI} 21 \mathrm{~cm}$ line is not detected at that velocity. The metallicity could actually be much higher, as high as that of the low-ionization clouds, but we have no way to place further constraints.

\subsection{Additional O I Cloud}

A model with three low-ionization clouds and one highionization cloud reproduces the observed spectrum very well except for the low-velocity side of O I, Fe II, and Si II. As noted in Section 3, we add an additional cloud at $\Delta v=-49 \mathrm{~km} \mathrm{~s}^{-1}$ (i.e., $V_{\odot}=161 \mathrm{~km} \mathrm{~s}^{-1}$ ) in order to fully produce the observed O I absorption. For this cloud, we can constrain the metallicity by the requirement that the optical depth of $\mathrm{H}$ I $21 \mathrm{~cm}$ at the line center must not exceed $\tau=0.025$. We obtain $\log \left(Z / Z_{\odot}\right) \geqslant-1.0$ for $\log U \geqslant-5.1$, and $\log \left(Z / Z_{\odot}\right) \geqslant-0.9$ for $\log U \leqslant-5.2$. The ionization parameter should be $\log U>-6.0$, below which $\mathrm{Mg}$ I would be overproduced. An upper limit on the ionization parameter of this component is -3.5 to avoid an overproduction of Si IV. We again see that nitrogen is deficient in this cloud by 0.6 dex. We give a sample of an acceptable model for this cloud, with $\log \left(Z / Z_{\odot}\right)=-1.0$ and $\log U=-5.1$ in Table 3 . However, we note that the parameters could instead be more similar to those of the other clouds.

\subsection{Our Best Model}

Table 3 lists the best parameters of our photoionization models: Column 1 is the optimized transition, Column 2 is the heliocentric velocity of the absorption line, Column 3 is relative velocity from the system center, Columns 4 and 5 are absorption optical depth at the line center and spin temperature measured from the $\mathrm{HI}$

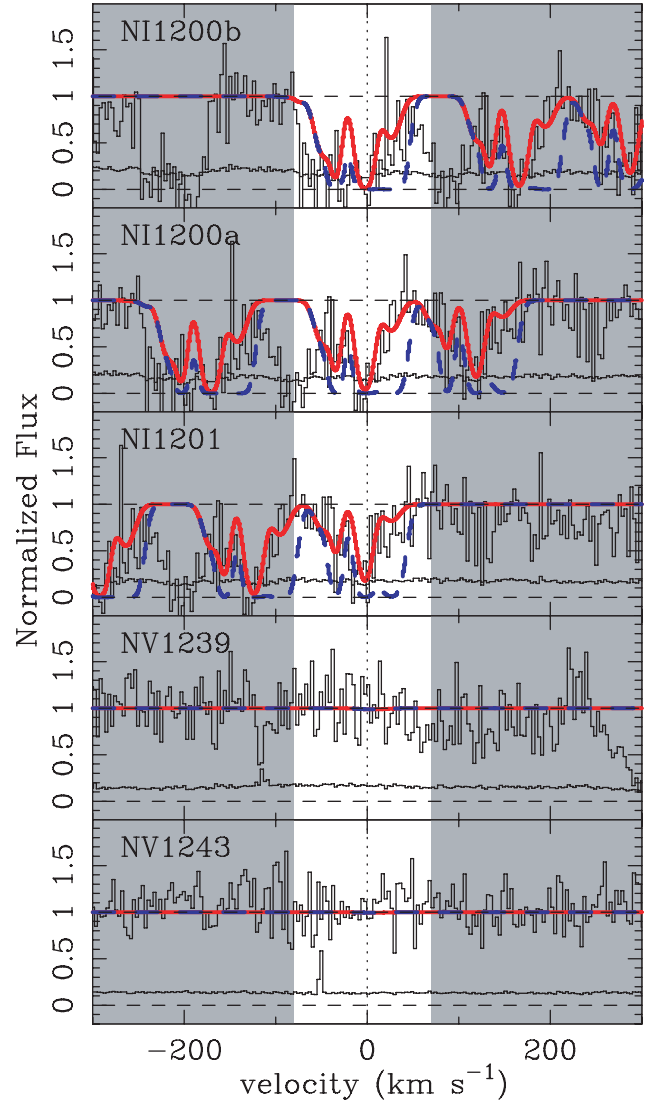

Figure 9. Comparison of the model spectra with nitrogen deficiency (solid red line) and without nitrogen deficiency (dashed blue line). Obviously, the model with solar abundance pattern does not reproduce the observed N I absorption lines. There is no large differences in the $\mathrm{N} \mathrm{v}$ doublet at this ionization parameter because of their very weak strengths. Unshaded parts are the regions of the absorber in the Magellanic Bridge.

(A color version of this figure is available in the online journal.)

$21 \mathrm{~cm}$ emission line (only for $\mathrm{HI} 21 \mathrm{~cm}$ lines), Columns 6 and 7 are the best Voigt-profile fit values of column density and Doppler parameter, Columns 8 and 9 are the best-model parameters (assuming a solar abundance pattern) of metallicity and ionization parameter, Column 10 is gas temperature from our model, Columns 11 and 12 are the gas volume density and the thickness of the absorber assuming a plane-parallel structure, and Column 13 notes changes in the nitrogen abundance (compared to the Solar abundance pattern) that are necessary to reproduce the observed spectrum. The synthesized spectrum, using the best-model parameters, is overplotted on the observed spectrum for each transition in Figure 8. If we compare the models with/without nitrogen deficiency to the observed spectrum, it is obvious that the nitrogen deficiency is necessary, as shown in Figure 9. A summary of how specific transitions were used to constrain $\log U$ and $\log \left(Z / Z_{\odot}\right)$ for the MB system is given in Table 5. We also compare the equivalent widths of the observed and the modeled spectra in Table 2, and confirmed they are in good agreement.

\subsection{Effects from Additional Radiation Sources}

Because this absorber is located in the MB, close to the MW and the LMC, the radiation field around it could be enhanced by contributions from these galaxies, as mentioned in Section 4.2. We perform photoionization analysis using the various radiation fields listed in Table 4. Because the purpose of this analysis is to 
Table 5

Model Constraints for the Magellanic Bridge Systems

\begin{tabular}{|c|c|c|c|c|}
\hline $\begin{array}{l}\text { Cloud } \\
\text { (1) }\end{array}$ & $\begin{array}{c}\text { Parameter } \\
\text { (2) }\end{array}$ & $\begin{array}{c}\text { Constraint } \\
\text { (3) }\end{array}$ & $\begin{array}{l}\text { Line } \\
(4)\end{array}$ & $\begin{array}{c}\text { Condition } \\
\text { (5) }\end{array}$ \\
\hline \multirow[t]{4}{*}{$\mathrm{HI}_{\mathrm{I}}\left(V_{\odot}=207 \mathrm{~km} \mathrm{~s}^{-1}\right)$} & $\log U$ & $>-6.0$ & C I & To avoid overproduction \\
\hline & & $<-4.8$ & $\mathrm{C} \mathrm{I}$ & To avoid underproduction \\
\hline & $\log Z$ & $\geqslant-0.5$ & $\mathrm{~S}_{\text {II }}$ & To avoid underproduction \\
\hline & & $\leqslant-0.5^{a}$ & Ni II & To avoid overproduction \\
\hline \multirow{4}{*}{$\mathrm{HI}\left(V_{\odot}=236 \mathrm{~km} \mathrm{~s}^{-1}\right)$} & $\log U$ & $>-6.1$ & $\mathrm{C} \mathrm{I}$ & To avoid overproduction \\
\hline & & $<-5.0^{b}$ & Si II & To avoid underproduction \\
\hline & $\log Z$ & $>-0.8$ & Si II & To avoid underproduction \\
\hline & & $<-0.6^{a}$ & Ni II & To avoid overproduction \\
\hline \multirow[t]{4}{*}{$\mathrm{HI}_{\mathrm{I}}\left(V_{\odot}=176 \mathrm{~km} \mathrm{~s}^{-1}\right)$} & $\log U$ & $>-6.0^{c}$ & C I & To avoid overproduction \\
\hline & & $<-5.0^{c}$ & Si II, O I & To avoid underproduction \\
\hline & $\log Z$ & $>-1.0$ & Si II & To avoid underproduction \\
\hline & & $<-0.7$ & Mg I, Ni II, C I & To avoid overproduction \\
\hline \multirow[t]{3}{*}{$\operatorname{SiIV}\left(V_{\odot}=217 \mathrm{~km} \mathrm{~s}^{-1}\right)$} & $\log U$ & $>-2.7$ & C IV & To avoid underproduction \\
\hline & & $<-2.4$ & C IV & To avoid overproduction \\
\hline & $\log Z$ & $>-4.0^{d}$ & H I $21 \mathrm{~cm}$ & To avoid overproduction \\
\hline \multirow[t]{3}{*}{$\mathrm{O}_{\mathrm{I}}\left(V_{\odot}=161 \mathrm{~km} \mathrm{~s}^{-1}\right)$} & $\log U$ & $>-6.0$ & Mg I & To avoid overproduction \\
\hline & & $<-3.5$ & Si IV & To avoid overproduction \\
\hline & $\log Z$ & $\geqslant-1.0^{d}$ & H I $21 \mathrm{~cm}$ & To avoid overproduction \\
\hline
\end{tabular}

Notes.

${ }^{a}$ Because nickel is strongly depleted on to the dust, this upper limit on the metallicity can be softened once the depletion is considered.

$\mathrm{b}$ This constraint is valid if we assume $\log \left(Z / Z_{\odot}\right)=-0.7$.

${ }^{\mathrm{c}}$ This constraint is valid if we assume $-1.0<\log \left(Z / Z_{\odot}\right)<-0.7$.

${ }^{\mathrm{d}}$ No upper limit can be placed because the corresponding H I $21 \mathrm{~cm}$ line is not detected with $>1 \sigma$ detection limit.

examine how the shape of radiation field affects the results of the photoionization modeling, we always use the best-fit parameters from Table 3. We compare the model spectra using various incident radiations to the observed spectrum in Figure 10. On the observed spectrum, we do not see any significant differences between the results for the different radiation field models, except for C I $\lambda 1329$, for which the model is slightly improved if we take a radiation from the LMC into account. However, the quality of spectrum around this line is not very high. Apparently the same fit parameters are still acceptable for the alternative radiation fields. However, once we estimate the line parameters of various transitions in the strongest component at $\Delta v=-3 \mathrm{~km} \mathrm{~s}^{-1}$ when using these incident fields, there are noticeable differences for some transitions (i.e., C IV, $\mathrm{N}$ III, $\mathrm{Al}$ III, and Si IV) that are very weak and/or positioned at low $\mathrm{S} / \mathrm{N}$ regions. This is because the number of hydrogen ionizing photon is basically same by our assumption (because we assume the same ionization parameter); however, the ionizing photons of some ions (especially ions with low ionization potentials) would be increased significantly once the additional radiation is considered. Although the ionization parameter that we infer is the same for these alternative radiation fields, the number of ionizing photons is substantially increased so that this same ionization parameter corresponds to a higher density. For the $\Delta v=-3 \mathrm{~km} \mathrm{~s}^{-1}$ component, the density has increased from $0.56 \mathrm{~cm}^{-3}$ to $19 \mathrm{~cm}^{-3}$, and the size decreased from $25 \mathrm{pc}$ to $0.72 \mathrm{pc}$ in the most extreme case. The other clouds are similarly smaller, with the Si IV cloud reduced in size to $0.57 \mathrm{kpc}$ under the more intense LMC radiation field. Since the differences in the ionization parameters that we infer are so small between the different models, it is not practical to distinguish between the different possible radiation fields using these data, and thus the inferred densities and sizes are uncertain. Hereafter, we discuss the results using only the EGB radiation, as summarized in Table 3.

\section{DISCUSSION}

\subsection{Origin of Metal Enrichment in the MB}

There have been three scenarios posed to explain the current chemical composition of the MB. Tidal stripping from the SMC, purported to explain the origin of the MB (Gardiner \& Noguchi 1996) would imply a chemical composition similar to the SMC itself, or perhaps slightly more metal-poor if the MB is preferentially formed from material drawn from the comparatively pristine outskirts of the SMC. The presence of any chemical gradient in the SMC is not yet well defined, but the recent results of Carrera et al. (2008) suggest it may be nonnegligible. A galactic wind from the SMC could enrich the MB with metals, and such a wind would likely be enhanced in alpha elements, given the composition of supernova-driven winds in other dwarf galaxies where such signatures are observed several $\mathrm{kpc}$ from their origin (Martin et al. 2002; Strickland et al. 2004). The final possibility is in situ enrichment from stars formed in the MB (Demers \& Battinelli 1998). Given the $200 \mathrm{Myr}$ age of the MB (Gardiner \& Noguchi 1996), there has been ample time for several generations of the most massive B stars to evolve and contribute their nucleosynthetic products to their surroundings. Some combinations of these processes are likely to play a role, but the balance of these has not yet been determined.

Rolleston et al. (1999) found an underabundance $(\sim 0.5 \mathrm{dex})$ in the light metals of a B-type star in the MB, DGIK 975, compared to the SMC HiI regions. Similarly, metallicity of the interstellar medium in the MB toward a young star, DI 1388 , was measured to be $\sim 0.2$ dex lower than the SMC H II regions (Lehner et al. 2008). The sightline toward our target, PKS 0312-770, has an angular separation of $\sim 4.1$ from DI 1388 , which corresponds to about $\sim 4.4 \mathrm{kpc}$ in physical scale at the distance of the SMC $(d \sim 60 \mathrm{kpc})$. However, we have found that the metallicity in the MB toward PKS 0312-770 is higher than that measured toward DI 1388 by $\sim 0.5 \mathrm{dex}$, and even $\sim 0.1-$ 


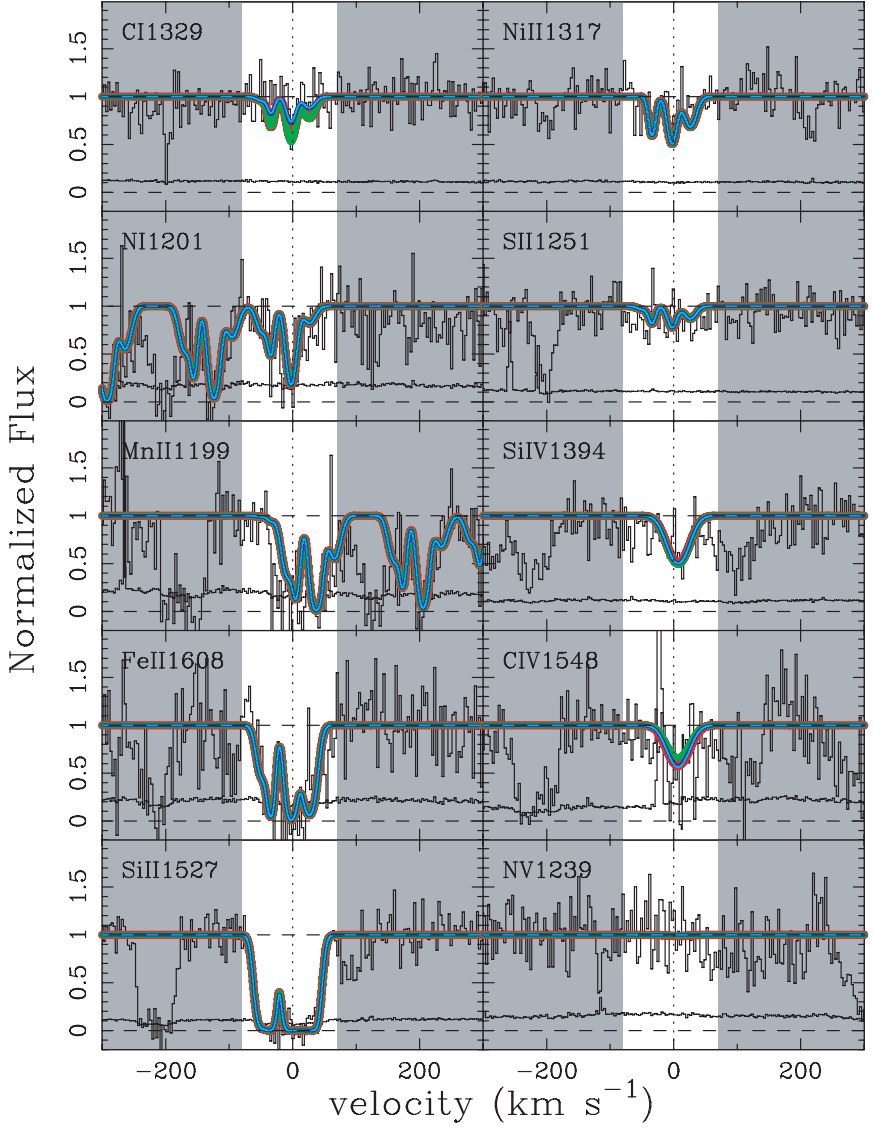

Figure 10. Comparison of the model spectra using different incident radiation fields: (a) only extra-galactic background radiation (EGR; red), (b) EGR plus radiation from the $\mathrm{MW}$ at a distance of $D=50 \mathrm{kpc}$ from the $\mathrm{MW}$ (green), (c) EGB, MW radiation, plus radiation from the LMC with a $15 \%$ escape ratio as a minimum flux model (thick blue), and (d) EGB, the MW radiation, plus radiation from the $\mathrm{LMC}$ with a $30 \%$ escape ratio as a maximum flux model (thin blue). Unshaded parts are the regions of the absorber in the Magellanic Bridge. (A color version of this figure is available in the online journal.)

0.2 dex more metal-rich than the SMC H II regions. What is the source of this difference? One possible idea is that the sightline toward PKS 0312-770 is not mixed with metal-poor gas, like the sightline toward DI 1388. If this is the case, absorbers with lower metallicities should have higher total hydrogen column densities. Such a trend (i.e., a gradual increase in metallicity with decreasing hydrogen column density) has already been pointed out for various objects at higher redshift, including galaxies, (sub)DLA systems, and the intergalactic medium (e.g., Boisse et al. 1998; Péroux et al. 2003; York et al. 2006; Misawa et al. 2008).

There are observational results that support the scenario above. Column densities of sulfur (a modestly dust-depleted element) are similar between these two sightlines. Each of the three clouds toward PKS 0312-770 (with $\log N\left(\mathrm{~S} /\left[\mathrm{cm}^{-2}\right]\right.$ )is 14.2, 14.4, and 14.2 $)^{12}$ has a similar column density to the total $\left(\log N\left(\mathrm{~S} /\left[\mathrm{cm}^{-2}\right]\right)=14.35\right)$ in DI 1388. However, the PKS 0312-770 clouds have total hydrogen column densities

\footnotetext{
12 We fitted the H I 21-cm absorption profile with three components. The total S II column density $\left(\log \left(S_{\text {II }}\right) \sim 14.75\right)$ is slightly lower than that measured in Lehner et al. (2008) because we assume $S_{\text {II }} \lambda 1254$ is blended with an unrelated line to avoid over-absorption of S II $\lambda 1251$, for which Lehner et al. (2008) applied the apparent optical depth (AOD) method directly.
}

$\left(\log N\left(\mathrm{H} /\left[\mathrm{cm}^{-2}\right]\right)=19.6,19.6,19.7\right)^{13} 0.6$ dex smaller than the total in DI $1388\left(\log N\left(\mathrm{H} /\left[\mathrm{cm}^{-2}\right]\right)=20.2\right)$. This implies a higher metallicity and less dilution in PKS 0312-770. These results are consistent with the scenario above, i.e., metal-enriched gas flowed out toward the MB from the SMC almost isotropically, but the MB absorbers toward PKS 0312-770 were only weakly diluted by metal-free material, while the absorber toward DI 1388 is significantly diluted.

\subsection{Nitrogen Deficiency in the $M B$}

The overall metallicity and the chemical abundance pattern in the MB can be used to infer the origin of this inter-Cloud material. Hambly et al. (1994) and Rolleston et al. (1999) measured $\mathrm{He}, \mathrm{O}, \mathrm{Si}, \mathrm{Mg}$, and $\mathrm{N}$ abundances for several early $\mathrm{B}$ stars in the $\mathrm{MB}$, assuming that their composition reflects the present-day interstellar medium (ISM), and concluded that these stars were 0.5 dex more metal-poor than SMC stars. These authors suggested that the MB stars formed from a mixture of SMC and unenriched gas. Dufour (1984) analyzed the H II region $\mathrm{He}, \mathrm{C}, \mathrm{N}, \mathrm{O}$, and $\mathrm{Si}$ abundances for the $\mathrm{SMC}$, finding that the overall metallicity, as measured by the alpha elements $\mathrm{O}$ and $\mathrm{Si}$, is $\sim 0.5$ dex more metal-poor than the Sun. The N/Si ratio in H II regions is 0.5 dex lower than the Sun, consistent with the trend observed in other low-metallicity dwarf galaxies (e.g., Kobulnicky \& Skillman 1996; Nava et al. 2006). We summarize these measurements from the literature along with ours in Table 6. Locations of the MB stars are also shown in Figure 1.

Figure 11 shows the abundance ratio $\log (\mathrm{N} / \mathrm{Si})$ versus $\log (\mathrm{Si} /$ $\mathrm{H})$ for the aforementioned measurements from Table 6 , assuming a solar abundance pattern if another element (instead of silicon) is used to estimate these parameters. The figure includes our 3-velocity components toward PKS 0312-770 (open circles), the Rolleston et al. (1999) MB stars (filled stars), the gas toward the MB star DI 1388 (Lehner et al. 2008; open star), the SMC star AV 304 (open square), the Sun (solar symbol), the range of SMC H II regions (filled square), the dwarf galaxies from Nava et al. (2006; crosses; values computed from $\mathrm{O}$ abundance measurements assuming a solar $\mathrm{Si} / \mathrm{O}$ ratio), and damped Ly $\alpha$ systems from Henry \& Prochaska (2007; dots). The abscissa reflects the overall alpha element abundance of the systems in question and shows that the metallicities of the SMC star AV 304, the MB stars, and the MB gas toward PKS 0312-770 are generally consistent with the range of metallicities in SMC H II regions. The MB star DGIK 975 is a possible exception, appearing $\sim 0.5$ dex more metal poor than the rest of these measurements. Figure 3 of Rolleston et al. (1999) shows that this star also lies the furthest from the SMC, in a low $\mathrm{H}$ I column density region equidistant between the SMC and LMC.

The N/Si ratio shown on the ordinate of Figure 11 is a measure of the chemical enrichment timescale. $\mathrm{Si}$ is synthesized in massive stars and returned to the ISM through supernovae on timescales of $\sim 10 \mathrm{Myr}^{14}$ Nitrogen, by contrast, is thought

\footnotetext{
13 Note that neutral hydrogen column densities toward DI 1388 and each of the three clouds toward PKS $0312-770$ are almost the same $\left(\log N_{\mathrm{H}_{\mathrm{I}}} \sim 19.6\right)$, but the former is considerably more ionized $\left(\log N_{\mathrm{H} \mathrm{II}} \sim 20.0\right)$ than the latter $\left(\log N_{\mathrm{H} \text { II }} \sim 17.7\right)$.

14 However, there is the possibility that $\alpha$-elements released by supernovae remain for an extended period in hot $10^{6} \mathrm{~K}$ bubbles and require several hundred Myr to mix with ambient galactic material (Tenorio-Tagle 1996; Kobulnicky \& Skillman 1997).
} 
Table 6

Comparison of Abundance Patterns ${ }^{\mathrm{a}}$

\begin{tabular}{|c|c|c|c|c|c|c|}
\hline \multirow{2}{*}{ Region } & \multicolumn{5}{|c|}{$\log ($ element/H) } & \multirow[b]{2}{*}{$\begin{array}{r}\text { ref. }^{b} \\
(7) \\
\end{array}$} \\
\hline & $\begin{array}{l}\mathrm{C} \\
(2) \\
\end{array}$ & $\begin{array}{l}\mathrm{N} \\
(3)\end{array}$ & $\begin{array}{l}\mathrm{O} \\
(4) \\
\end{array}$ & $\begin{array}{l}\mathrm{Si} \\
(5) \\
\end{array}$ & $\begin{array}{c}S \\
(6)\end{array}$ & \\
\hline The Sun & $-3.61 \pm 0.04$ & $-4.17 \pm 0.11$ & $-3.31 \pm 0.05$ & $-4.46 \pm 0.05$ & $-4.80 \pm 0.05$ & 1 \\
\hline SMC H II region & $\begin{array}{l}-4.47 \pm 0.06 \\
-4.84 \pm 0.04\end{array}$ & $\begin{array}{l}-5.41 \pm 0.08 \\
-5.54 \pm 0.12\end{array}$ & $\begin{array}{l}-3.95 \pm 0.08 \\
-3.98 \pm 0.08\end{array}$ & $\begin{array}{c}-5.30 \pm 0.2 \\
\ldots\end{array}$ & $\begin{array}{l}-5.58 \pm 0.11 \\
-5.51 \pm 0.14\end{array}$ & $\begin{array}{l}2 \\
3\end{array}$ \\
\hline SMC star (AV 304) & -5.15 & $-5.11 \pm 0.17$ & $-3.90 \pm 0.16$ & $-5.27 \pm 0.02$ & $\ldots$ & 4 \\
\hline Bridge star (DI 1162) & $-4.94 \pm 0.33$ & -5.30 & $-4.00 \pm 0.13$ & $-5.19 \pm 0.06$ & $\ldots$ & 4 \\
\hline Bridge star (DGIK 975) & $-5.32 \pm 0.25$ & -5.26 & $-3.96 \pm 0.21$ & $-5.78 \pm 0.15$ & $\ldots$ & 4 \\
\hline Bridge gas $\left(V_{\odot}=176 \mathrm{~km} \mathrm{~s}^{-1}\right)$ & -4.61 to -4.31 & -6.37 to -5.37 & -4.31 to -4.01 & -5.46 to -5.16 & -5.74 to -5.44 & 5 \\
\hline Bridge gas $\left(V_{\odot}=207 \mathrm{~km} \mathrm{~s}^{-1}\right)$ & -4.11 & -5.37 to -5.17 & -3.81 & -4.96 & -5.24 & 5 \\
\hline Bridge gas $\left(V_{\odot}=236 \mathrm{~km} \mathrm{~s}^{-1}\right)$ & -4.41 to -4.21 & -6.47 to -5.87 & -4.11 to -3.91 & -5.26 to -5.06 & -5.54 to -5.34 & 5 \\
\hline Bridge gas (toward DI 1388) & $\ldots$ & $-5.29 \pm 0.11$ & $-4.30+0.13-0.11$ & $-5.45+0.14-0.12^{c}$ & $\ldots$ & 6 \\
\hline
\end{tabular}

Notes.

a All abundance patterns are measured from the observed spectra directly, except for our results for which we estimate them based on the photoionization model.

b 1: Lodders (2003), 2: Kurt et al. (1999), 3: Dufour (1984), 4: Rolleston et al. (1999), 5: this paper, 6: Lehner et al. (2008)

${ }^{c}$ Converted from $\log (\mathrm{O} / \mathrm{H})$, assuming solar abundance pattern.

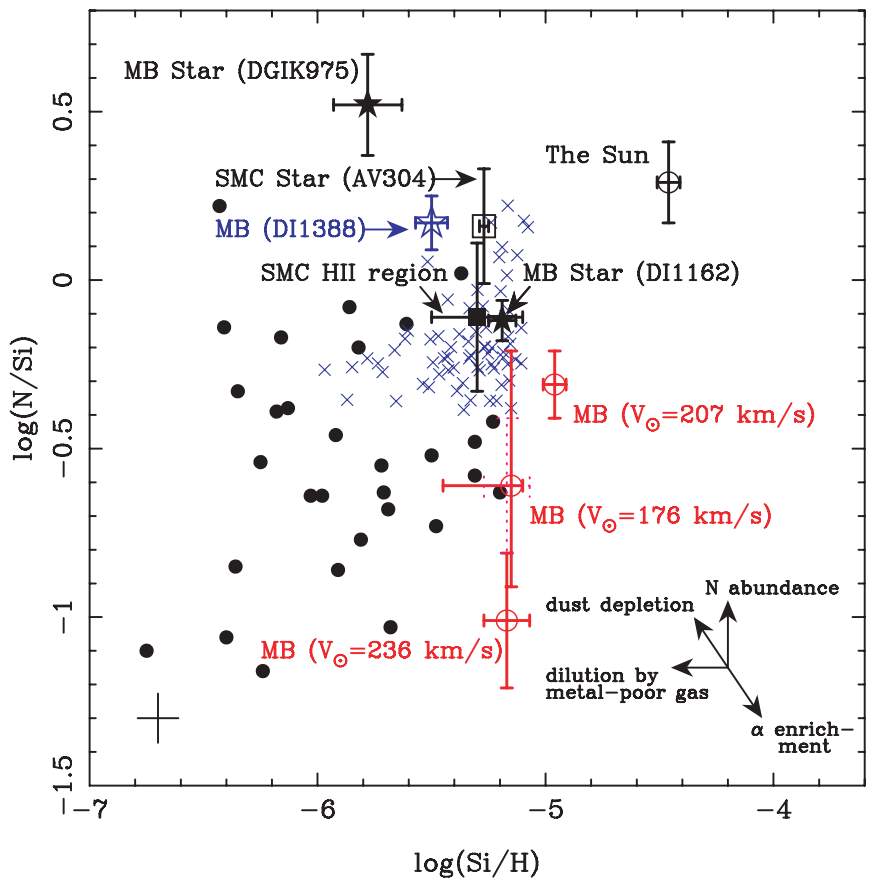

Figure 11. $\log (\mathrm{N} / \mathrm{Si})$ versus $\log (\mathrm{Si} / \mathrm{H})$ of three absorption components in the Magellanic Bridge toward PKS 0312-770, compared to those of DLA systems (dots; Henry \& Prochaska 2007), blue compact galaxies (blue cross; Nava et al. 2006) in which oxygen abundance was converted to silicon abundance using the solar abundance ratio (Holweger 2001; Allende Prieto et al. 2001), and other SMC/MB objects from Table 6 (error bars). Error bars shown in the bottom left are average uncertainties of the DLA systems in each direction. The dotted error bar denotes the $1 \sigma$ error in our determination of the abundance ratio for the $\mathrm{H}$ component at $V_{\odot}=236 \mathrm{~km} \mathrm{~s}^{-1}$, if we reduce the nitrogen abundance by -1.0 dex instead of -1.4 dex that is still consistent with the observed spectrum. The four possible evolutionary vectors are also shown in the bottom right. We do not plot components at $V_{\odot}=161 \mathrm{~km} \mathrm{~s}^{-1}$ and $217 \mathrm{~km} \mathrm{~s}^{-1}$, because we cannot place any meaningful constraints on their abundance patterns.

(A color version of this figure is available in the online journal.)

to be produced in most galaxies by low- and intermediatemass stars (LIMS) and released on timescales of $>100 \mathrm{Myr}$ (van den Hoek \& Groenewegen 1997; Marigo 2001; Renzini \&
Voli 1981). ${ }^{15}$ Thus, the $\mathrm{N} / \mathrm{Si}$ ratio may drop during prolonged starbursts and rise during prolonged periods of quiescence, functioning as a kind of "clock," marking the time since the most recent major episode of star formation (Edmunds \& Pagel 1978; Pantelaki 1988; Garnett 1990; Kobulnicky \& Skillman 1998). Henry \& Prochaska (2007) used this scenario to model the chemical evolution of DLA systems in the N/Si versus $\mathrm{Si} / \mathrm{H}$ plane and found that they could reproduce the properties of most DLA systems as a function of two variables: the star formation efficiency and the age of the system. They concluded that small ages (i.e., less than $250 \mathrm{Myr}$ ) are required to produce the low $\mathrm{N} / \mathrm{Si}$ systems, while high star formation efficiencies lead to higher $\mathrm{Si} / \mathrm{H}$ and lower N/Si ratios. However, a number of other processes may contribute to shaping the chemical evolution of a system. The arrows in Figure 11 show qualitatively the four possible evolutionary vectors attributed to $\mathrm{N}$ enrichment (vertically upward), $\alpha$ enrichment (toward the lower right), dust depletion (toward upper left), and dilution by metal-poor gas (leftward).

Figure 11 shows that the $207 \mathrm{~km} \mathrm{~s}^{-1}$ component toward PKS $0312-770$ is $0.3-0.4$ dex more metal-rich than the bulk of the SMC and other MB stars. This component also exhibits an $\mathrm{N} / \mathrm{Si}$ ratio that is $0.2-0.3$ dex lower than the SMC stars and $\mathrm{H}$ II regions. This departure is consistent with a composition consisting of SMC material augmented by a small amount of $\alpha$ enrichment from supernovae. None of the MB stars in Figure 11 nor the ISM probed toward the MB star DI 1388 share this abundance pattern. ${ }^{16}$ The PKS $0312-770$ line of sight is therefore the first MB location with a metallicity that is similar to, or perhaps slightly higher than, the SMC at large.

Figure 11 shows that the absorbing component at $236 \mathrm{~km} \mathrm{~s}^{-1}$ has a metallicity similar to the SMC and most MB stars, but the N/Si ratio is about 1.0 dex lower. ${ }^{17}$ Such a

\footnotetext{
15 See, however, Ackerman et al. (2004); Spite et al. (2005); Pettini et al. (2008) for evidence that massive stars may dominate $\mathrm{N}$ production at very low metallicities, $Z<0.01 Z_{\odot}$.

16 Hot stars in the MB could be affected by additional production of nitrogen. 17 Normalization of the spectrum may be performed incorrectly to the low signal-to-noise $(\sim 5)$ spectrum region around $\mathrm{N}_{\mathrm{I}}$ lines. If we reduce nitrogen abundance by only -1.0 dex from the solar abundance pattern (i.e., same as the $176 \mathrm{~km} \mathrm{~s}^{-1}$ component), $\mathrm{N}_{\mathrm{I}} \lambda 1200 \mathrm{~b}$ and $\mathrm{N}_{\mathrm{I}} \lambda 1201$ would be reproduced better (see Figure 12).
} 
low $\mathrm{N} / \mathrm{Si}$ ratio places this material among the most $\mathrm{N}$-deficient DLA systems and suggests a nucleosynthetic history dominated by $\alpha$-producing massive stars. In the Henry \& Prochaska (2007) models, such a low $\mathrm{N}$ abundance at such high metallicity can only be achieved by very efficient and very recent star formation such that massive stars dominate the mass-averaged nucleosynthetic contribution with virtually no contribution from longer lived N-producing stars. Given the presence of B stars in the MB (e.g., Rolleston et al. 1999), some supernova activity during the $<200 \mathrm{Myr}$ lifetime of the MB is likely. In situ enrichment appears a plausible explanation for this component on the basis of chemical cloud. However, it is also possible that a Si enrichment of ambient SMC material plus dilution from putative metal-poor halo gas could also explain this data point (i.e., a combination of vectors that together drive evolution in Figure 11 in a downward vertical direction from the SMC H II regions composition).

Finally, the $176 \mathrm{~km} \mathrm{~s}^{-1}$ component has an $\mathrm{Si} / \mathrm{H}$ ratio consistent with the SMC and $236 \mathrm{~km} \mathrm{~s}^{-1}$ component, but the uncertainties on the $\mathrm{N} / \mathrm{Si}$ ratio are too large to place meaningful constraints on the origin of this material. Moreover, abundance pattern would also be strongly affected by dust depletion and ionization conditions, which can only be explored with very high $\mathrm{S} / \mathrm{N}$ spectrum. Therefore, this component may either be $\mathrm{N}$-deficient or it may be consistent with the SMC H II regions.

\subsection{Dust Depletion in the $M B$}

In addition to various absorption lines we have detected in the HST/STIS spectrum, Smoker et al. (2005) also detected the Ca II $\mathrm{K}$ line in their medium resolution $(R=6000)$ optical spectra of seven quasars behind the MB including our target (Smoker et al. 2005). By comparing the total column densities of $\mathrm{Ca}$ II and $\mathrm{H}_{\mathrm{I}}$ (measured from H I $21 \mathrm{~cm}$ emission line), they found that the abundance ratio of $\mathrm{Ca}$ II to $\mathrm{H} \mathrm{I}$ in the $\mathrm{MB}$ is systematically higher than that of Galactic gas by a factor of $\sim 0.5$ dex. Smoker et al. (2005) proposed possible scenarios for this difference, such as the higher ionization condition of the hydrogen gas, and weaker dust depletion in the MB. To test the scenario, a higher resolution spectrum will be necessary to deblend an unresolved Ca II K profile into multiple components (as we did for other transitions in the UV spectrum) in order to constrain the photoionization model.

In Section 5, we found the best-model parameters. However, there is still an ambiguity due to the possible effects of dust, which would lead to different inferred physical conditions. Our data were not of sufficient quality to measure dust depletion, but the estimated low gas temperature of the three H I clouds $\left(T_{\text {gas }}\right.$ $<1000 \mathrm{~K}$ ) would imply that dust grains could survive in the absorber. Moreover, the Ca II $\mathrm{K}$ absorption strength, expected from our best model ( $\left.W_{\text {rest }}=0.99 \AA\right)$, is 7 times greater than the observed value toward PKS 0312-770 ( $W_{\text {rest }}=0.14 \AA$; Smoker et al. 2005). This also implies that the absorber contains substantial amounts of dust, because calcium is one of the most severely depleted elements (Savage \& Sembach 1996). Actually, Lehner et al. (2008) suggested Si and Fe in the MB toward DI 1388 are depleted to dust by factors of -0.45 and -0.61 dex, respectively, although the condition could be different toward PKS 0312-770. If depletion onto dust is significant, then the $\mathrm{Si}$ abundances and the implied metallicities become even larger, making this sightline significantly more metal-enriched than the SMC itself.

\subsection{Comparison with DLA Systems at high-z}

DLAs are characterized by high $\mathrm{H}_{\mathrm{I}}$ column densities (i.e., $\log N\left(\mathrm{H}_{\mathrm{I}}\right) \geqslant 2 \times 10^{20} \mathrm{~cm}^{-3}$ ) and low metallicities; i.e., $\log \left(Z / Z_{\odot}\right) \sim 0.1-0.01$ (e.g., Pettini et al. 1997; Prochaska et al. 2003). DLAs are also known to have lower nitrogen abundance relative to $\alpha$ elements, compared to the solar abundance pattern (e.g., Pettini et al. 2002). DLAs provide plentiful information on the physical condition in gas clouds in the ancient universe that can never be traced by stellar objects. However, so far DLA absorbers have not been identified clearly, especially at higher redshift ${ }^{18}$, although at least elliptical galaxies are probably ruled out (Calura et al. 2003). Thus, it is quite helpful to find local counterparts of those systems and study their properties in detail.

With respect to $\mathrm{H}$ I column density, the $\mathrm{MB}$ absorbers toward PKS 0312-770, whose total column density is just below the criterion to be classified as a DLA system, could be analogs of high- $z$ DLA systems. However, their nitrogen abundance relative to $\alpha$ elements (e.g., $[\mathrm{N} / \mathrm{Si}]$ ) tend to be small and positioned at the lowest end of the [N/Si] distribution of DLA systems in Figure 11. Particularly, the nitrogen abundance of the $V_{\odot}=236$ cloud is very small compared to that expected for DLA systems and blue compact galaxies of a similar metallicity. The origin of this difference should be understood before using the MB absorbers as local counterparts of high- $z$ DLA systems.

The nitrogen deficiency may be linked to the synthesis process. It is already known that nitrogen is produced by LIMS $\left(M / M_{\odot} \leqslant 8\right)$, while $\alpha$ elements are produced by massive stars. For DLA systems with low nitrogen (hereafter, low nitrogen DLAs: LNDLAs), there have been two possible scenarios presented (Henry \& Prochaska 2007 and references therein), i.e., (1) they are in early production stages of nitrogen released from LIMS (delay scenario; Pettini et al. 2002), and (2) they have an intrinsically flattened or truncated initial mass function (IMF) with fewer LIMS (reduction scenario; Prochaska et al. 2002). However, as summarized in Henry \& Prochaska (2007), neither scenario can explain the nitrogen deficiency of LNDLAs perfectly; the former cannot reproduce a possible bimodality of $[\mathrm{N} / \alpha]$ distribution of DLAs (Prochaska et al. 2002; Centurión et al. 2003), while the latter produces too much iron compared with the observed amount (Lanfranchi \& Matteucci 2003).

Thus, we have not yet identified the origin of the nitrogen deficiency. Nonetheless, it is likely that the production history of nitrogen is not the same between the SMC and the MB. The IMF of a stellar association NGC 602 in the SMC (a single power law with a slope of $\Gamma \sim-1.2$ for $M / M_{\odot}=$ 1-45; Schmalzl et al. 2008) is quite similar to the IMF in the solar neighborhood $\left(\Gamma \sim-1.35\right.$ for $M / M_{\odot}=0.4-10$; Salpeter 1955). This means that the abundance patterns of the MW and the SMC are expected to be similar, while they can be different from that of the MB, whose IMF is not necessarily the same. The MB probably has a local star-forming history independent of the nearby star-forming regions (i.e., the LMC and the SMC), which is consistent with the observed results that the young stars in the MB are not old enough to have escaped from the SMC based on their peculiar motions.

If the $\mathrm{MB}$ absorbers are indeed local counterparts of high- $z$ DLAs, our results would have interesting implications: (1) DLA systems along our sightlines to the background quasars do not

\footnotetext{
18 Although several DLA host galaxies have been detected (e.g., Rao \& Turnshek 2000; Djorgovski et al. 1996; Möller et al. 2004) there is still considerable ambiguity about what part of the galaxy and its environment is responsible for the actual DLA.
} 


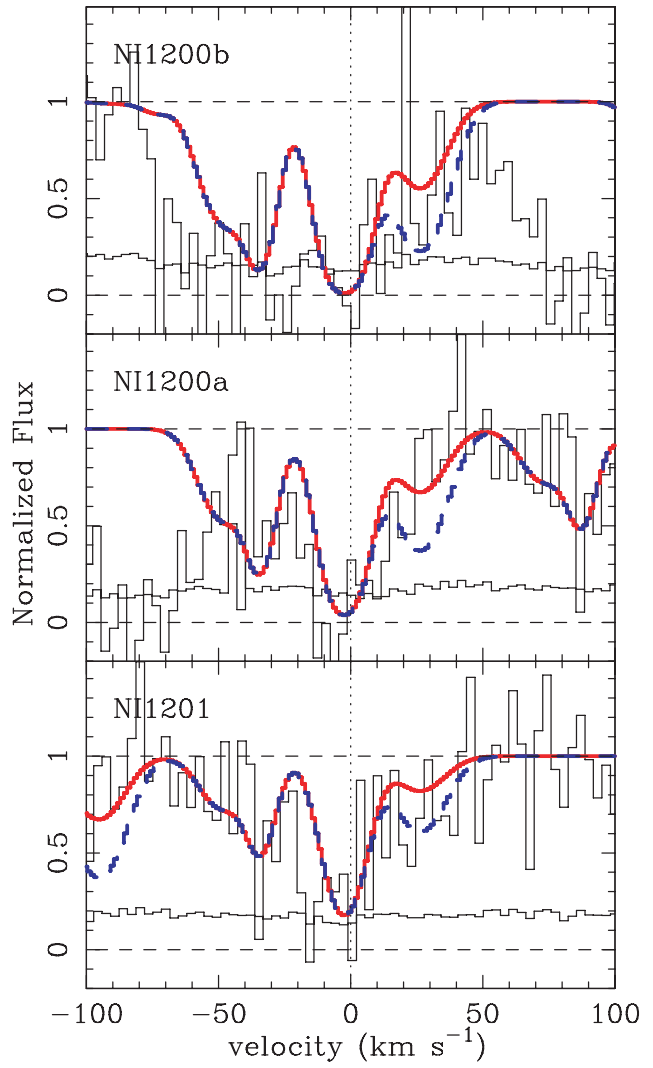

Figure 12. Close up velocity plot around three detected $\mathrm{N}$ I absorption lines within $\pm 100 \mathrm{~km} \mathrm{~s}^{-1}$ of the system center. Solid lines represent a model in which we decrease nitrogen abundance of the $\mathrm{H}_{\mathrm{I}}$ component at $V_{\odot}=237 \mathrm{~km}$ $\mathrm{s}^{-1}$ by 1.4 dex compare to the other elements (i.e., our best model in Table 3 ), while dashed lines are for the same model but with the nitrogen abundance reduced by 1.0 dex. If the continuum fitting around N I 1200a is incorrect, this latter model would reproduce absorption features of the other two Ni lines much better. Moreover, this weaker nitrogen deficiency is more reasonable if we compare to those of the other two $\mathrm{H}$ I components at $V_{\odot}=176$ and $207 \mathrm{~km}$ $\mathrm{s}^{-1}$.

(A color version of this figure is available in the online journal.)

necessarily represent global properties of absorbing structures; and (2) the large scatter of metallicity and $[\mathrm{N} / \alpha]$ values could be due to the internal gradient of each DLA absorber, and they are different from place to place along different sightlines go through. For example, we would underestimate the global metallicity (and nitrogen abundance) of the Magellanic Clouds if our line of sight went through only the MB. Moreover, there might be metallicity gradient in the MB itself-higher in the SMC wing than in the remaining parts of the MB (Lehner et al. 2008 and references therein). As for DLAs, Ellison et al. (2005) and Chen et al. (2005) already suggested that there could be a metallicity gradient as a function of a distance from the galactic center. Ellison et al. (2005) and Wolfe et al. (2003) also proposed that the DLA region is sampling a lower metallicity than the star-forming regions. Thus, high- $z$ DLA systems also may have complex internal structures like the Magellanic Clouds and their neighborhood.

This research was funded by the National Science Foundation (NSF) under grant AST 04-07138 and by NASA under grant NAG5-6399. T.M. acknowledges support from the Special Postdoctoral Research Program of RIKEN. This work was supported by NASA through grant NAG5-10770 to H.A.K. B.P.W. was supported by HST grant HST-R-10984.01-A and
NASA ADP grant NNX07AH42G. J.B.H. is supported by a Federation Fellowship from the Australian Research Council. We thank Nicolas Lehner and the anonymous referee for their valuable comments. T.M. acknowledges support from the Special Postdoctoral Research Program of RIKEN and the Sumitomo Foundation (070380).

\section{REFERENCES}

Ackerman, C. J., Carigi, L., Nissen, P. E., Pettini, M., \& Asplund, M. 2004, A\&A, 414, 931

Allende Prieto, C., Lambert, D. L., \& Asplund, M. 2001, ApJ, 556, L63

Battinelli, P., \& Demers, S. 1992, AJ, 104, 1458

Bergeron, J., \& Boissé, P. 1991, A\&A, 243, 344

Bland-Hawthorn, J., Sutherland, R., Agertz, O., \& Moore, B. 2007, ApJ, 670, L109

Bland-Hawthorn, J., \& Maloney, P. R. 2002, in ASP Conf. Proc. 254, Extragalactic Gas at Low Redshift, ed. J. S. Mulchaey \& J. Stocke (San Francisco, CA: ASP), 267

Bland-Hawthorn, J., \& Maloney, P. R. 1999, ApJ, 510, L33

Boisse, P., Le Brun, V., Bergeron, J., \& Deharveng, J.-M. 1998, A\&A, 333, 841

Braun, R., \& Walterbos, R. A. M. 1992, ApJ, 386, 120

Brown, T., et al. 2002, in HST STIS Data Handbook, version 4.0, ed. B. Mobasher (STScI: Baltimore), 131

Calura, F., Matteucci, F., \& Vladilo, G. 2003, MNRAS, 340, 59

Carrera, R., Gallart, C., Aparicio, A., Costa, E., Méndez, R. A., \& Noël, N. E. D. 2008, AJ, 136, 1039

Centurión, M., Molaro, P., Vladilo, G., Péroux, C., Levshakov, S. A., \& D'Odorico, V. 2003, A\&A, 403, 55

Chen, H.-W., Kennicutt, R. C. Jr., \& Rauch, M. 2005, ApJ, 620, 703

Churchill, C. W., \& Charlton, J. C. 1999, AJ, 118, 59

Churchill, C. W., Vogt, S. S., \& Charlton, J. C. 2003, AJ, 125, 98

Churchill, C. W., \& Vogt, S. S. 2001, AJ, 122, 679

Demers, S., \& Battinelli, P. 1998, AJ, 115, 154

Dickey, J. M., Marx-Zimmer, M., Düsterberg, C., Meb, U., Stanimirovic, S., Staveley-Smith, L., \& Kobulnicky, H. A. 1999, in IAU Symp. 190, New Views of the Magellanic Clouds, ed. Y.-H. Chu et al. (San Francisco, CA: IAU), 45

Dickey, J. M., Mebold, U., Marx, M., Amy, S., Haynes, R. F., \& Wilson, W. 1994, A\&A, 289, 357

Djorgovski, S. G., Pahre, M. A., Bechtold, J., \& Elston, R. 1996, Nature, 382, 234

Dufour, R. J. 1984, in IAU Symp. 108, Structure and Evolution of the Magellanic Clouds, ed. S. van den Bergh \& K. S. de Boer (Dordrecht: Reidel), 353

Edmunds, M. G., \& Pagel, B. E. J. 1978, MNRAS, 185, 78

Ellison, S. L., Kewley, L. J., \& Mallén-Ornelas, G. 2005, MNRAS, 357, 354

Ferland, G. J., Korista, K. T., Verner, D. A., Ferguson, J. W., Kingdon, J. B., \& Verner, E. M. 1998, PASP, 110, 761

Fox, A. J., Wakker, B. P., Savage, B. D., Tripp, T. M., Sembach, K. R., \& Bland-Hawthorn, J. 2005, ApJ, 630, 332

Gardiner, L. T., \& Noguchi, M. 1996, MNRAS, 278, 191

Gardiner, L. T., Sawa, T., \& Fujimoto, M. 1994, MNRAS, 266, 567

Garnett, D. R. 1990, ApJ, 363, 142

Giandoni, S. 2005, Master thesis, New Mexico State University, NM, USA

Grondin, L., Demers, S., \& Kunkel, W. E. 1992, AJ, 103, 1234

Guhathakurta, P., \& Reitzel, D. B. 1998, Galactic Halos, 136, 22

Haardt, F., \& Madau, P. 2001, arXiv:astro-ph/0106018

Haardt, F., \& Madau, P. 1996, ApJ, 461, 20

Hambly, N. C., Dufton, P. L., Keenan, F. P., Rolleston, W. R. J., Howarth, I. D., \& Irwin, M. J. 1994, A\&A, 285, 716

Harris, J. 2007, ApJ, 658, 345

Harries, T. J., Hilditch, R. W., \& Howarth, I. D. 2003, MNRAS, 339, 157

Henry, R. B. C., \& Prochaska, J. X. 2007, PASP, 119, 962

Hindman, J. V., Kerr, F. J., \& McGee, R. X. 1963, Aust. J. Phys., 16, 570

Holweger, H. 2001, in AIP Conf. Proc. 598, Joint SOHO/ACE Workshop "Solar and Galactic Composition," ed. R. F. Wimmer-Schweingruber (San Francisco, CA: ASP), 23

Irwin, M. J., Kunkel, W. E., \& Demers, S. 1985, Nature, 318, 160

Irwin, M. J., Demers, S., \& Kunkel, W. E. 1990, AJ, 99, 191

Kalberla, P. M. W., Burton, W. B., Hartmann, D., Arnal, E. M., Bajaja, E., Morras, R., \& Poppel, W. G. L. 2005, A\&A, 440, 775

Kallivayalil, N., van der Marel, R. P., \& Alcock, C. 2006, ApJ, 652, 1213

Kobulnicky, H. 2000, HST Proposal, 4987

Kobulnicky, H. A., \& Dickey, J. M. 1999, AJ, 117, 908

Kobulnicky, H. A., \& Skillman, E. D. 1998, ApJ, 497, 601 
Kobulnicky, H. A., \& Skillman, E. D. 1997, ApJ, 489, 636

Kobulnicky, H. A., \& Skillman, E. D. 1996, ApJ, 471, 211

Kurt, C. M., Dufour, R. J., Garnett, D. R., Skillman, E. D., Mathis, J. S., Peimbert, M., Torres-Peimbert, S., \& Ruiz, M.-T. 1999, ApJ, 518, 246

Lanfranchi, G. A., \& Matteucci, F. 2003, MNRAS, 345, 71

Lehner, N., Howk, J. C., Keenan, F. P., \& Smoker, J. V. 2008, ApJ, 678, 219

Lehner, N. 2002, ApJ, 578, 126

Lehner, N., Sembach, K. R., Dufton, P. L., Rolleston, W. R. J., \& Keenan, F. P. 2001, ApJ, 551, 781

Li, A., Misselt, K. A., \& Wang, Y. J. 2006, ApJ, 640, L151

Lodders, K. 2003, ApJ, 591, 1220

Lu, L., Sargent, W. L. W., Barlow, T. A., Churchill, C. W., \& Vogt, S. S. 1996, ApJS, 107, 475

Mallouris, C., et al. 2001, ApJ, 558, 133

Marigo, P. 2001, A\&A, 370, 194

Martin, C. L., Kobulnicky, H. A., \& Heckman, T. M. 2002, ApJ, 574, 663

Mathewson, D. S., \& Ford, V. L. 1984, in IAU Symp. 108, Structure and Evolution of the Magellanic Clouds, ed. S. van den Bergh \& K. S. de Boer (Dordrecht: Reidel), 125

Mebold, U., Duesterberg, C., Dickey, J. M., Staveley-Smith, L., \& Kalberla, P. 1997, ApJ, 490, L65

Misawa, T., Charlton, J. C., \& Narayanan, A. 2008, ApJ, 679, 220

Mizuno, N., Muller, E., Maeda, H., Kawamura, A., Minamidani, T., Onishi, T., Mizuno, A., \& Fukui, Y. 2006, ApJ, 643, L107

Möller, P., Fynbo, J. P. U., \& Fall, S. M. 2004, A\&A, 422, L33

Muller, E., Staveley-Smith, L., \& Zealey, W. J. 2003, MNRAS, 338, 609

Murai, T., \& Fujimoto, M. 1980, PASJ, 32, 581

Nava, A., Casebeer, D., Henry, R. B. C., \& Jevremovic, D. 2006, ApJ, 645, 1076

Nishiyama, S., et al. 2007, ApJ, 658, 358

Pagel, B. E. J., Edmunds, M. G., Fosbury, R. A. E., \& Webster, B. L. 1978, MNRAS, 184, 569

Pantelaki, I. 1988, PhD thesis, Rice University

Péroux, C., Dessauges-Zavadsky, M., D’Odorico, S., Kim, T.-S., \& McMahon, R. G. 2003, MNRAS, 345, 480
Pettini, M., Zych, B. J., Steidel, C. C., \& Chaffee, F. H. 2008, MNRAS, 385, 2011

Pettini, M., Ellison, S. L., Bergeron, J., \& Petitjean, P. 2002, A\&A, 391, 21

Pettini, M., Smith, L. J., King, D. L., \& Hunstead, R. W. 1997, ApJ, 486, 665

Piatek, S., Pryor, C., \& Olszewski, E. W. 2008, AJ, 135, 1024

Prochaska, J. X., Gawiser, E., Wolfe, A. M., Castro, S., \& Djorgovski, S. G. 2003, ApJ, 595, L9

Prochaska, J. X., Henry, R. B. C., O’Meara, J. M., Tytler, D., Wolfe, A. M., Kirkman, D., Lubin, D., \& Suzuki, N. 2002, PASP, 114, 933

Putman, M. E., et al. 1998, Nature, 394, 752

Renzini, A., \& Voli, M. 1981, A\&A, 94, 175

Rao, S. M., \& Turnshek, D. A. 2000, ApJS, 130, 1

Rolleston, W. R. J., Dufton, P. L., McErlean, N. D., \& Venn, K. A. 1999, A\&A 348,728

Salpeter, E. E. 1955, ApJ, 121, 161

Savage, B. D., \& Sembach, K. R. 1996, ARA\&A, 34, 279

Schmalzl, M., Gouliermis, D. A., Dolphin, A. E., \& Henning, T. 2008, ApJ, 681,290

Shapley, H. 1940, Harv. Coll. Obs. Bull., 914, 8

Smoker, J. V., Keenan, F. P., Thompson, H. M. A., Brüns, C., Muller, E., Lehner, N., Lee, J.-K., \& Hunter, I. 2005, A\&A, 443, 525

Spite, M., et al. 2005, A\&A, 430, 655

Strickland, D. K., Heckman, T. M., Colbert, E. J. M., Hoopes, C. G., \& Weaver, K. A. 2004, ApJS, 151, 193

Tenorio-Tagle, G. 1996, AJ, 111, 1641

van den Hoek, L. B., \& Groenewegen, M. A. T. 1997, A\&AS, 123, 305

Welty, D. E., Lauroesch, J. T., Blades, J. C., Hobbs, L. M., \& York, D. G. 2001, ApJ, 554, L75

Wolfe, A. M., Gawiser, E., \& Prochaska, J. X. 2003, ApJ, 593, 235

York, D. G., et al. 2006, MNRAS, 367, 945

Zonak, S. G., Charlton, J. C., Ding, J., \& Churchill, C. W. 2004, ApJ, 606, 196 